\title{
Generalizing Consistency and other Constraint Properties to Quantified Constraints
}

\author{
LUCAS BORDEAUX \\ Microsoft Research \\ MARCO CADOLI \\ Università di Roma "La Sapienza" \\ TONI MANCINI \\ Università di Roma "La Sapienza"
}

\begin{abstract}
Quantified constraints and Quantified Boolean Formulae are typically much more difficult to reason with than classical constraints, because quantifier alternation makes the usual notion of solution inappropriate. As a consequence, basic properties of Constraint Satisfaction Problems (CSP), such as consistency or substitutability, are not completely understood in the quantified case. These properties are important because they are the basis of most of the reasoning methods used to solve classical (existentially quantified) constraints, and one would like to benefit from similar reasoning methods in the resolution of quantified constraints.

In this paper, we show that most of the properties that are used by solvers for CSP can be generalized to quantified CSP. This requires a re-thinking of a number of basic concepts; in particular, we propose a notion of outcome that generalizes the classical notion of solution and on which all definitions are based. We propose a systematic study of the relations which hold between these properties, as well as complexity results regarding the decision of these properties. Finally, and since these problems are typically intractable, we generalize the approach used in CSP and propose weaker, easier to check notions based on locality, which allow to detect these properties incompletely but in polynomial time.
\end{abstract}

Categories and Subject Descriptors: F4.1 [Mathematical Logic and Formal Languages]: Logic and Constraint Programming

General Terms: Algorithms

Additional Key Words and Phrases: Constraint Satisfaction, Quantified Constraints, Quantified Boolean Formulae

Authors'address: Lucas Bordeaux, Microsoft Research Ltd, Roger Needham Building, J J Thomson Avenue, Cambridge CB3 0FB, United Kingdom.

Marco Cadoli and Toni Mancini, Università di Roma La Sapienza, Dipartimento di Informatica e Sistemistica, Via Salaria 113, 00198 Rome, Italy.

A preliminary version of this paper appears in Proc. of the 20th National Conf. on Artificial Intelligence, published by the American Association of Artificial Intelligence [Bordeaux et al. 2005]. The current paper is a revised and extended version that includes proofs of all results.

Permission to make digital/hard copy of all or part of this material without fee for personal or classroom use provided that the copies are not made or distributed for profit or commercial advantage, the ACM copyright/server notice, the title of the publication, and its date appear, and notice is given that copying is by permission of the ACM, Inc. To copy otherwise, to republish, to post on servers, or to redistribute to lists requires prior specific permission and/or a fee.

(C) 2018 ACM 1529-3785/18/1000-0001 $\$ 5.00$ 


\section{INTRODUCTION}

\subsection{Quantified Constraints}

Quantified Constraint Satisfaction Problems (QCSP) have recently received increasing attention from the Artificial Intelligence community [Bordeaux and Monfroy 2002; Börner et al. 2003; Chen 2004a; 2004b; Mamoulis and Stergiou 2004; Gent et al. 2004; Gent et al. 2005; Verger and Bessière 2006; Benedetti et al. 2007; Bordeaux and Zhang 2007]. A large number of solvers are now available for Quantified Boolean Formulae (QBF), which represent the particular case of QCSP where the domains are Boolean and the constraints are clauses, see e.g., [Buening et al. 1995; Cadoli et al. 1999; Cadoli et al. 2002; Rintanen 1999] for early papers on the subject, and [Benedetti 2004; Zhang 2006; Samulowitz et al. 2006; Samulowitz and Bacchus 2006] for descriptions of state-of-the-art techniques for QBF. The reason behind this trend is that QCSP and QBF are natural generalizations of CSP and SAT that allow to model a wide range of problems not directly expressible in these formalisms, and with applications in Artificial Intelligence and verification.

\subsection{Reasoning with Quantified Constraints}

Quantified constraints are typically much more difficult to reason with than classical constraints. To illustrate this difficulty, let us start by an example of property we would like to characterize formally, and let us suggest why a number of naive attempts to define this property are not suitable. Consider the formula:

$$
\phi: \quad \forall x \in[3,10] . \exists y \in[1,15] . x=y .
$$

We would like to "deduce" in a sense that $y \in[1,10]$ or, in other words, that the values $[11,15]$ are inconsistent for $y$. Such a property will in particular be useful to a search-based solver: if this inconsistency is revealed, then the solver can safely save some effort by skipping the branches corresponding to the values $y \in[11,15]$.

A first attempt to define this notion of consistency would be to use an implication and to say, for instance, that value $a$ is consistent for $y$ iff $\phi \rightarrow(y=a)$. But there is clearly a problem with this approach since the occurrence of $y$ on the right-handside of the implication is unrelated to its occurrences in formula $\phi$, which fall under the scope of a quantifier. One may attempt to circumvent this problem by putting the implication under the scope of the quantifiers, and to say, for instance, that $a$ is consistent for $y$ iff $\forall x \in[3,10]$. $\exists y \in[1,15] .(x=y) \rightarrow(y=a)$. But with this definition any value would in fact be consistent, even $y=17$. This is because for every $x$, we have a value for $y$ that falsifies the left-hand side of the implication, thereby making the implication true.

Another approach that looks tempting at first but is also incorrect is to say that $a$ is inconsistent for $y$ iff the formula obtained by fixing the domain of $y$ to $\{a\}$ is false. With this definition we would deduce that all values $a \in[1,15]$ are inconsistent w.r.t. variable $y$, since the formula $\forall x \in[3,10] . \exists y \in[a, a] . x=y$ is false in each and every case. Other variants of these definitions can be considered, but one quickly gets convinced that there is simply no natural way to define consistency, or any other property like interchangeability, using implications or instantiations. To define these notions properly in the case of quantified constraints, we need a new framework, which is what this paper proposes.

ACM Transactions on Computational Logic, Vol. V, No. N, October 2018. 


\subsection{Overview of our Contributions}

This paper shows that the definitions of consistency, substitutability, and a wider range of CSP properties can be generalized to quantified constraints. Note that all our definitions and results also hold for the particular case of Quantified Boolean Formulas. These definitions, presented in Section 3, are based on a simple gametheoretic framework and in particular on the new notion of outcome which we identify as a key to define and understand all QCSP properties. We then classify these properties in Section 4 by studying the relationships between them (e.g., some can be shown to be stronger than others). We investigate the simplifications allowed by these properties in Section 5, and we characterize the complexity of their associated decision problem in Section 6. Since, as these complexity results show, determining whether any property holds is typically intractable in general, we investigate the use of the same tool which is used in classical CSP, namely local reasoning, and we propose in Section 7 local versions of these properties that can be decided in polynomial time. Concluding comments follow in Section 8. We start (Section 2) by introducing some material on QCSP.

\section{QUANTIFIED CONSTRAINT SATISFACTION PROBLEMS}

In this section, we present all the definitions related to QCSP, as well as some "game-theoretic" material.

\subsection{Definition of QCSP}

Let $\mathbb{D}$ be a finite set. Given a finite set $V$ of variables, a $V$-tuple $t$ with components in $\mathbb{D}$, is a mapping that associates a value $t_{x} \in \mathbb{D}$ to every $x \in V$; a $V$-relation over $\mathbb{D}$ is a set of $V$-tuples with components in $\mathbb{D}$.

Definition 1. A Quantified Constraint Satisfaction Problem (QCSP) is a tuple $\phi=\langle X, Q, D, C\rangle$ where: $X=\left\{x_{1}, \ldots, x_{n}\right\}$ is a linearly ordered, finite set of variables; $Q$ associates to each variable $x_{i} \in X$ a quantifier $Q_{x_{i}} \in\{\forall, \exists\} ; D$ associates to every variable $x_{i} \in X$ a domain $D_{x_{i}} \subseteq \mathbb{D}$; and $C=\left\{c_{1}, \ldots c_{m}\right\}$ is a finite set of constraints, each of which is a $V$-relation with components in $\mathbb{D}$ for some $V \subseteq X$.

\subsubsection{Notation}

- The notation $\prod_{x \in V} D_{x}$, where $V \subseteq X$ is a subset of variables, will denote a Cartesian product of domains, i.e., the set of $V$-tuples $t$ that are such that $t_{x} \in D_{x}$ for each $x \in V$.

- The notation $t[x:=a]$, where $t$ is an $X$-tuple, $x \in X$ is a variable and $a \in \mathbb{D}$ is a value, will be used for instantiation, i.e., it denotes the tuple $t^{\prime}$ defined by $t_{x}^{\prime}=a$ and $t_{y}^{\prime}=t_{y}$ for each $y \in X \backslash\{x\}$.

- The notation $\left.t\right|_{U}$, where $t$ is a $V$-tuple and $U \subseteq V$ is a subset of its variables, will denote the restriction of $t$ to $U$, i.e., the $U$-tuple $t^{\prime}$ such that $t_{x}^{\prime}=t_{x}$ for each $x \in U$. (Note that $t$ is undefined on every $y \in V \backslash U$.)

We use the following shorthands to denote the set of existential (resp. universal) variables, the set of variables of index $\leq j$, and the sets of existential/universal 
variables of index $\leq j$ :

$$
\begin{array}{ll} 
& X_{j}=\left\{x_{i} \in X \mid i \leq j\right\} \\
E=\left\{x_{i} \in X \mid Q_{x_{i}}=\exists\right\} & E_{j}=E \cap X_{j} \\
A=\left\{x_{i} \in X \mid Q_{x_{i}}=\forall\right\} & A_{j}=A \cap X_{j}
\end{array}
$$

2.1.2 Satisfaction, Solutions and Truth of a QCSP. Given a QCSP $\phi=\langle X, Q, D, C\rangle$ as in Definition 1, an $X$-tuple $t$ is said to satisfy the set of constraints $C$ if $\left.t\right|_{V} \in c$ for each $V$-relation $c \in C$. The set of $X$-tuples satisfying all constraints of $\phi$ is called the set of solutions to $C$ and is denoted by sol ${ }^{\phi}$.

Although QCSPs are defined in a form that closely follows the traditional definition of CSPs, the most immediate way to define their semantics is to use rudimentary logic with equality. (We shall see in the next section that we can in a second step forget about the logic and think alternatively in terms of tuples and functions when this is more convenient.) A QCSP $\langle X, Q, D, C\rangle$ represents a logical formula whose vocabulary includes $n$ names for the variables (for convenience, we simply denote these names as $\left.x_{1} \ldots x_{n}\right)$ and $m$ names for the constraints $\left(c_{1} \ldots c_{m}\right)$. The formula is defined as:

$$
F: \quad Q_{x_{1}} x_{1} \in D_{x_{1}} \ldots Q_{x_{n}} x_{n} \in D_{x_{n}}\left(F_{1} \wedge \cdots \wedge F_{m}\right) .
$$

where each $F_{i}$ is obtained from the corresponding $V$-relation $c_{i}$ : let $\left\{y_{1}, . ., y_{p}\right\}=V$, then $F_{i}$ is simply the formula $c_{i}\left(y_{1}, . ., y_{p}\right)$, i.e., we apply the name of the constraint to the right argument list. Each $D_{x_{i}}$ explicitly lists the values specified in the QCSP definition, for instance $\forall x \in\{a, b\} . \phi$ is a shorthand for $\forall x .(x=a \vee x=b) \rightarrow \phi$.

Let $I$ be the interpretation function that associates to each constraint name the corresponding relation; the QCSP is said to be true if formula $F$ is true in the domain $\mathbb{D}$ and w.r.t. the interpretation $I$, i.e., iff $\langle\mathbb{D}, I\rangle \models F$.

\subsection{Game-Theoretic Material}

Quantifier alternation is best understood using an "adversarial" or "game-theoretic" viewpoint, where two players interact. One of them is allowed to choose the values for the existential variables, and its aim is to ultimately make the formula true, while the other assigns the universal variables and aims at falsifying it. We introduce several definitions leading to our central notion of outcome, which will be shown to shed light on the definition of properties in the next section. Our presentation of the basic game-theoretic material is inspired from [Chen 2004b], who uses a similar notion of winning strategy.

The following QCSP (written using the usual, self-explanatory logical notation rather than in the form of a tuple $\langle X, Q, D, C\rangle$ ) will be used to illustrate the notions throughout this sub-section:

$$
\begin{aligned}
\exists x_{1} \in[1,10] . & \forall x_{2} \in[1,10] . \exists x_{3} \in[1,10] . \\
\forall x_{4} \in[1,10] . \exists x_{5} \in[1,10] . & x_{1}+x_{2}+x_{3}+x_{4}+x_{5}=30
\end{aligned}
$$

This formula can be thought of as a game between two players assigning, respectively, the odd and even variables. The players draw in turn between 1 and 10 sticks from a heap containing originally 30 sticks; the player who takes the last stick wins.

ACM Transactions on Computational Logic, Vol. V, No. N, October 2018. 


\subsubsection{Strategies. The first notion we need is the notion of strategy:}

Definition 2. A strategy is a family $\left\{s_{x_{i}} \mid x_{i} \in E\right\}$ where each $s_{x_{i}}$ is a function of signature $\left(\prod_{y \in A_{i-1}} D_{y}\right) \rightarrow D_{x_{i}}$.

In other words, a strategy defines for each existential variable $x_{i}$ a function that specifies which value to pick for $x_{i}$ depending on the values assigned to the universal variables that precede it. Note in particular that, if the first $k$ variables of the problem are quantified existentially, we have for every $i \leq k$ a constant $s_{x_{i}} \in D_{x_{i}}$ which defines which value should directly be assigned to variable $x_{i}$.

EXAMPLE 1. A strategy for the QCSP (1) can be defined by $s_{x_{1}}()=8 ; s_{x_{3}}$ associates to every $\left\{x_{2}\right\}$-tuple $t$ the value $s_{x_{3}}(t)=11-t_{x_{2}}$ and $s_{x_{5}}$ associates to every $\left\{x_{2}, x_{4}\right\}$-tuple $t$ the value $s_{x_{5}}(t)=11-t_{x_{4}}$. This strategy specifies that we first draw 8 sticks, then for the next moves we shall draw 11 minus what the opponent just drew.

2.2.2 Scenarios. The tuple of values that will eventually be assigned to the variables of the problem depends on two things: 1) the strategy we have fixed a priori, and 2) the sequence of choices of the "adversary", i.e., the values that are assigned to the universal variables. Given a particular strategy, a number of potential scenarios may therefore arise, depending on what the adversary will do. These scenarios are defined as follows:

Definition 3. The set of scenarios of a strategy $s$ for a QCSP $\phi$, denoted $\operatorname{sce}^{\phi}(s)$, is the set of tuples $t \in \prod_{x \in X} D_{x}$ such that, for each $x_{i} \in E$, we have:

$$
t_{x_{i}}=s_{x_{i}}\left(\left.t\right|_{A_{i-1}}\right)
$$

In other words, the values for the existential variables are determined by the strategy in function of the values assigned to the universal variables preceding it. There is no restriction, on the contrary, on the values assigned to universal variables: this reflects the fact that we model the viewpoint of the existential player, and the adversary may play whatever she wishes to play.

EXAMPLE 1. (Ctd.) An example of scenario for the strategy defined previously is the tuple defined by $x_{1}=8, x_{2}=4, x_{3}=7, x_{4}=1, x_{5}=10$. On the contrary, the tuple $x_{1}=8, x_{2}=4, x_{3}=7, x_{4}=1, x_{5}=5$ is not a scenario since the value 5 for $x_{5}$ does not respect what is specified by $s_{x_{5}}$.

2.2.3 Winning Strategies. Of particular interest are the strategies whose scenarios are all solutions. We call them winning strategies:

Definition 4. A strategy $s$ is a winning strategy for the QCSP $\phi$ if every scenario $t \in \operatorname{sce}^{\phi}(s)$ satisfies the constraints of $\phi$ (in other words: if $\operatorname{sce}^{\phi}(s) \subseteq \operatorname{sol}^{\phi}$ ).

We denote by WIN $^{\phi}$ the set of winning strategies of the QCSP $\phi$.

EXAMPLE 1. (Ctd.) In the strategy s defined in Example 1, any scenario $t$ is of the form $x_{1}=8, x_{2}=a, x_{3}=11-a, x_{4}=b, x_{5}=11-b$. As a result the sum always evaluates to $8+a+11-a+b+11-b=30$ and $s$ is therefore a winning strategy. In fact, this strategy is the only winning one; one can check, for instance, that the strategy $s^{\prime}$ defined by $s_{x_{1}}^{\prime}()=7 ; s_{x_{3}}^{\prime}(t)=7$ and $s_{x_{5}}^{\prime}(t)=7$ is not winning. 
The following proposition is essential in that it justifies the use of the gametheoretic approach ${ }^{1}$ :

Proposition 1. A QCSP is true (as defined in Section 2.1.2) iff it has a winning strategy.

2.2.4 Outcome. Whereas the preceding material is well-known and is used, for instance, in [Chen 2004b], we introduce the following new notion:

Definition 5. The set of outcomes of a QCSP $\phi$, denoted out ${ }^{\phi}$, is the set of all scenarios of all its winning strategies, i.e., it is defined as:

$$
\text { out }^{\phi}=\bigcup_{s \in \mathrm{WIN}^{\phi}} \operatorname{sce}^{\phi}(s)
$$

EXAMPLE 1. (Ctd.) Since our example has a unique winning strategy it is easy to characterise its set of outcomes: these are all the tuples of the form $x_{1}=8, x_{2}=$ $a, x_{3}=11-a, x_{4}=b, x_{5}=11-b$, with $a, b \in[1,10]$.

Outcomes are related to the classical notion of solution in the following way: in general any outcome satisfies the set of constraints $C$, so we have out ${ }^{\phi} \subseteq$ sol $^{\phi}$, and the equality out ${ }^{\phi}=$ sol $^{\phi}$ holds if all variables are existential. On the other hand let us emphasize the fact that not all solutions are necessarily outcomes in general: in our example the tuple $x_{1}=6, x_{2}=6, x_{3}=6, x_{4}=6, x_{5}=6$ is for instance a solution as it satisfies the unique constraint $\left(x_{1}+x_{2}+x_{3}+x_{4}+x_{5}=30\right)$. But there is no winning strategy whose set of scenarios includes this particular tuple, and it is therefore not an outcome.

The notion of outcome is a generalization of the notion of solution that takes into account the quantifier prefix of the constraints. Our claim in the following is that outcomes play a role as central for QCSP as the notion of solution does in CSP, and that most definitions can be based on this notion.

2.2.5 Summary of the notions and notations. To summarize, we have defined 3 sets of tuples $\left(\mathrm{sol}^{\phi}\right.$ : the set of solutions, $\operatorname{sce}^{\phi}(s)$ : the set of scenarios of strategy $s$, and out ${ }^{\phi}$ : the set of outcomes) and one set of strategies (WIN ${ }^{\phi}$ : the set of winning strategies). All the game-theoretic notions we have introduced are illustrated in Fig. 1, where we consider the QCSP represented by the logical formula:

$$
\exists x_{1} \in[2,3] \forall x_{2} \in[3,4] \exists x_{3} \in[3,6] . x_{1}+x_{2} \leq x_{3} .
$$

And and or labels on the nodes correspond to universal and existential quantifiers, respectively. The solutions are all triples $\left\langle x_{1}, x_{2}, x_{3}\right\rangle$ s.t. $x_{1}+x_{2} \leq x_{3}$. The only two winning strategies assign $x_{1}$ to 2 : one $\left(s_{1}\right)$ systematically assigns $x_{3}$ to 6 while the 2nd one $\left(s_{2}\right)$ assigns it to $x_{2}+2$ (note that each strategy is constrained to choose one unique branch for each existential node). The scenarios of $s_{1}$ and $s_{2}$ are therefore those indicated, while the set of outcomes of the QCSP is the union of the scenarios of $s_{1}$ and $s_{2}$ (also shown in bold line).

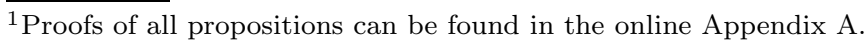

ACM Transactions on Computational Logic, Vol. V, No. N, October 2018. 


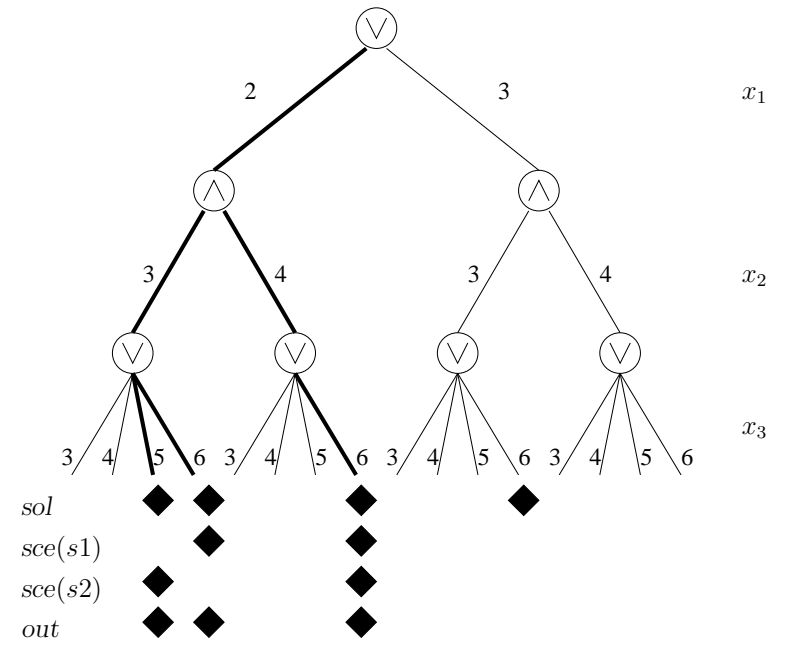

Fig. 1. A summary of the game-theoretic notions used in this paper.

\section{DEFINITIONS OF THE CSP PROPERTIES}

\subsection{Informal Definitions of the Properties}

A major part of the CSP literature aims at identifying properties of particular values of some variables. The goal is typically to simplify the problem by ruling out the possibility that a variable $x_{i}$ can be assigned to a value $a$. This can be done when one of the following properties holds, with respect to variable $x_{i}$ :

- Value $a$ is guaranteed not to participate in any solution: $a$ is inconsistent for $x_{i}$ [Mackworth 1977].

-Another value $b$ can replace $a$ in any solution involving it: $a$ is substitutable to $b$ for $x_{i}$ [Freuder 1991].

-All solutions involving $a$ can use another value instead: $a$ is removable for $x_{i}$ [Bordeaux et al. 2004].

On the contrary, some other properties give an indication that instantiating $x_{i}$ to $a$ is a good idea:

-All solutions assign value $a$ to variable $x_{i}: a$ is implied for $x_{i}$ [Monasson et al. 1999];

- We have the guarantee to find a solution with value $a$ on $x_{i}$, if a solution exists at all: $a$ is said to be fixable for $x_{i}$ [Bordeaux et al. 2004].

While all the preceding are properties of particular values, related properties of variables are also of interest:

- The value assigned to a variable $x_{i}$ is forced to a unique possibility: $x_{i}$ is determined.

- The value of variable $x_{i}$ is a function of the values of other variables: $x_{i}$ is dependent. 
- Whether a tuple is a solution or not does not depend on the value assigned to variable $x_{i}: x_{i}$ is irrelevant.

In this section, we propose generalizations of the definitions of the main CSP properties to quantified constraints. For the sake of homogeneity, we adopt the terminology used in the paper [Bordeaux et al. 2004] for the names of the properties.

We adopt a predicate notation and write, e.g., $p^{\phi}\left(x_{i}, a\right)$ for the statement "value $a$ has property $p$ for variable $x_{i}$ (in QCSP $\phi$ )". The superscript $\phi$ will be omitted in order to simplify the notation whenever there is no ambiguity regarding which QCSP is considered.

We present our definitions in two steps: Section 3.2 introduces the basic definitions, which we call deep definitions, for reasons that will become clear in the rest of this section. We then notice in Section 3.3 that the properties can be made more general, leading to our shallow definitions.

\subsection{Basic Definitions}

The first definitions we propose are identified by a $d$ prefix and qualified as "deep" when an ambiguity with the definitions in forthcoming Section 3.3 is possible. They are based on directly rephrasing the original CSP definitions, but using the notion of outcomes in place of solutions:

Definition 6. We define the properties of inconsistency, implication, deep fixability, deep substitutability, deep removability, deep interchangeability, determinacy, deep irrelevance and dependency, as follows, for all $x_{i} \in X, a, b \in D_{x_{i}}, V \subseteq X$ :

$$
\begin{aligned}
\text { inconsistent }\left(x_{i}, a\right) & \equiv \quad \forall t \in \text { out. } \quad t_{x_{i}} \neq a \\
\operatorname{implied}\left(x_{i}, a\right) & \equiv \quad \forall t \in \text { out. } t_{x_{i}}=a
\end{aligned}
$$

We note that the definition of consistency is equivalent to the one proposed in [Bordeaux and Monfroy 2002]; it is nevertheless expressed in a simpler and more elegant way that avoids explicitly dealing with And/Or trees. All other definitions are new.

EXAMPLE 2. Consider the QCSP:

$$
\exists x_{1} \in[2,3] \forall x_{2} \in[3,4] \exists x_{3} \in[3,6] . x_{1}+x_{2} \leq x_{3}
$$

ACM Transactions on Computational Logic, Vol. V, No. N, October 2018. 
(cf. Fig. 1). We have: inconsistent $\left(x_{1}, 3\right)$, inconsistent $\left(x_{3}, 3\right)$, inconsistent $\left(x_{3}, 4\right)$, $d$-substitutable $\left(x_{3}, 5,6\right), d$-fixable $\left(x_{3}, 6\right), d$-removable $\left(x_{3}, 5\right)$, and implied $\left(x_{1}, 2\right)$.

A choice we made in Definition 6 requires a justification: if we consider, for instance, fixability, one may think that a more general definition could be obtained

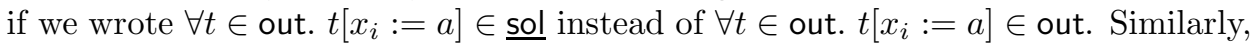
the question arises whether the other definitions that involve the set out in the righthand side of an implication (either implicitly or explicitly) could be strengthened be using the set sol instead. This is not the case: except for one property, namely determinacy, the modified definitions would actually be strictly equivalent:

Proposition 2. Deep fixability could equivalently be defined by the condition $\forall t \in$ out.t $\left[x_{i}:=a\right] \in$ sol; Deep substitutability could be equivalently defined by $\forall t \in$ out. $\left(t_{x_{i}}=a\right) \rightarrow\left(t\left[x_{i}:=b\right] \in\right.$ sol $)$; deep removability by $\forall t \in$ out. $\left(t_{x_{i}}=a\right) \rightarrow$ $\left(\exists b \neq a . t\left[x_{i}:=b\right] \in\right.$ sol $)$; and deep irrelevance by $\forall t \in$ out. $\forall b \in D_{x_{i}} . t\left[x_{i}:=b\right] \in$ sol.

This proposition will play a role in the proof of other results. Defining determinacy by $\forall t \in$ out. $\forall b \neq t_{x_{i}} . t\left[x_{i}:=b\right] \notin$ sol, instead of the definition we used. i.e., $\forall t \in$ out. $\forall b \neq t_{x_{i}} . t\left[x_{i}:=b\right] \notin$ out, would on the contrary give a slightly different notion: we note that in this case (because of the negation implicitly on the righthand side of the implication, i.e., $t\left[x_{i}:=b\right] \notin$ out), the definition would become weaker. For instance, in Fig. 1, we would not have determined $\left(x_{1}, 2\right)$ because the tuple $t=\langle 2,3,6\rangle$ is such that $t\left[x_{1}:=3\right] \in$ sol.

\subsection{Generalization: Shallow Definitions}

The previous definitions are correct in a sense that will be made formal in Section 5 . They are nevertheless overly restrictive in some cases, as the following example shows:

Example 3. Consider the QCSP:

$$
\forall x_{1} \in[1,2] \exists x_{2} \in[3,4] \exists x_{3} \in[4,6] . x_{1}+x_{2}=x_{3} .
$$

The winning strategies can make arbitrary choices for $x_{2}$ as long as they set $x_{3}$ to the value $x_{1}+x_{2}$, and the outcomes are the triples $\langle 1,3,4\rangle,\langle 1,4,5\rangle,\langle 2,3,5\rangle$, $\langle 2,4,6\rangle$. Note that for variable $x_{2}$, neither values 3 nor 4 are deep-fixable, and none is deep-substitutable to the other. This somehow goes against the intuition that we are indeed free to choose the value for $x_{2}$.

The reason why our previous definition did not capture this case is that it takes into account the values of the variables occurring after the considered variable: values 3 and 4 are interchangeable (for instance) only if the QCSPs resulting from these instantiations can be solved using the same strategy for all the subsequent choices - this is why we called these definitions deep (with a $d$ prefix). On the contrary, we can formulate shallow definitions of the properties, which accept value 4 as a valid substitute for 3 because in any sequence of choices leading to the possibility of choosing 3 for $x_{2}$, value 4 is also a valid option.

Definition 7. We define the properties of shallow fixability, substitutability, re- 
movability, interchangeability, and irrelevance, as follows:

$$
\begin{aligned}
& \text { s-fixable }\left(x_{i}, a\right) \equiv \\
& \forall t \in \text { out. } \exists t^{\prime} \in \text { out. }\left(\left.t\right|_{X_{i-1}}=\left.t^{\prime}\right|_{X_{i-1}} \wedge t_{x_{i}}^{\prime}=a\right) \\
& \text { s-substitutable }\left(x_{i}, a, b\right) \equiv \\
& \forall t \in \text { out. } t_{x_{i}}=a \rightarrow \\
& \quad \exists t^{\prime} \in \text { out. }\left(\left(\left.t\right|_{X_{i-1}}=\left.t^{\prime}\right|_{X_{i-1}}\right) \wedge\left(t_{x_{i}}^{\prime}=b\right)\right) \\
& \text { s-removable }\left(x_{i}, a\right) \equiv \\
& \forall t \in \text { out. } t_{x_{i}}=a \rightarrow \\
& \quad \exists t^{\prime} \in \text { out. }\left(\left.t\right|_{X_{i-1}}=\left.t^{\prime}\right|_{X_{i-1}} \wedge t_{x_{i}}^{\prime} \neq a\right) \\
& \text { s-interchangeable }\left(x_{i}, a, b\right) \equiv \\
& \text { s-substitutable }\left(x_{i}, a, b\right) \wedge \text { s-substitutable }\left(x_{i}, b, a\right) \\
& \text { s-irrelevant }\left(x_{i}\right) \equiv \\
& \forall t \in \text { out. } \forall b \in D_{x_{i}} . \\
& \quad \exists t^{\prime} \in \text { out. }\left(\left(\left.t\right|_{X_{i-1}}=\left.t^{\prime}\right|_{X_{i-1}}\right) \wedge\left(t_{x_{i}}^{\prime}=b\right)\right)
\end{aligned}
$$

One can check that with these definitions we handle Example 3 as expected:

Example 3. (Ctd.) Considering again the QCSP:

$$
\forall x_{1} \in[1,2] \exists x_{2} \in[3,4] \exists x_{3} \in[4,6] . x_{1}+x_{2}=x_{3},
$$

values 3 and 4 are shallow-interchangeable for variable $x_{2}$ (both values are also shallow-fixable, shallow-removable, and variable $x_{2}$ is in fact shallow-irrelevant). The reason is that for each outcome that assigns value 3 to $x_{2}$, there exists a tuple $t^{\prime}$ such that $t_{x_{1}}^{\prime}=t_{x_{1}}$ and $t_{x_{2}}^{\prime}=4$ (to $t=\langle 1,3,4\rangle$ corresponds $t^{\prime}=\langle 1,4,5\rangle$; to $\langle 2,3,5\rangle$ corresponds $\langle 2,4,6\rangle)$, and vice-versa.

This can be seen pictorially in Fig. 2. On the left-hand side, we see why values 3 and 4 are not (for instance) deep-interchangeable for $x_{2}$ : the outcomes (branches) going through these values are indeed different. Now on the right-hand side we see the viewpoint of the shallow definitions: the strategy is only considered up to variable $x_{2}$, and it is clear, then, that values 3 and 4 are interchangeable.

We last remark that the distinction we have introduced between deep and shallow only makes sense for a subset of the properties. It is easy to see, for instance, that a shallow definition of inconsistency would make no difference: this notion is defined by the statement $\forall t \in$ out. $t_{x_{i}} \neq a$, and this is equivalent to $\forall t \in$ out. $\left(\left.t\right|_{X_{i}}\right)_{x_{i}} \neq a$.

\section{RELATIONS BETWEEN THE PROPERTIES}

This section gives a number of results establishing the relations between the classes of properties (e.g., deep, shallow) and between the properties themselves (substitutability, determinacy, etc.). These results will also be used later (Section 5) to prove that our definitions are useful, in that they allow to simplify the considered QCSP while preserving some form of equivalence.

ACM Transactions on Computational Logic, Vol. V, No. N, October 2018. 


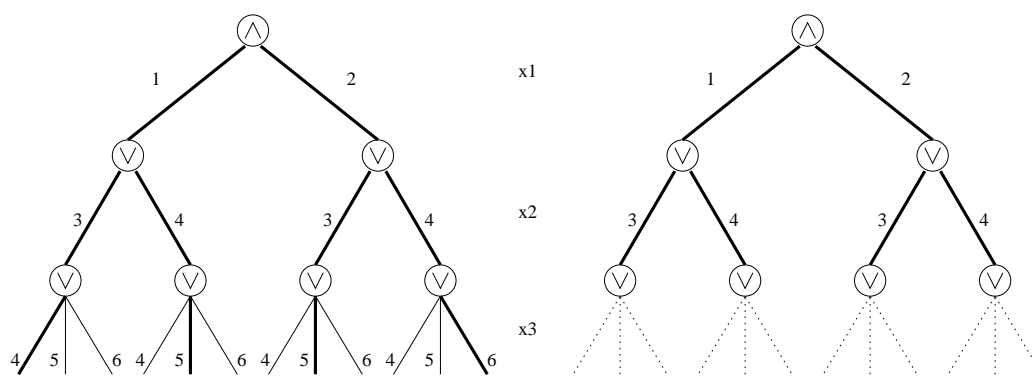

Fig. 2. Illustration of the notion of shallow properties, as opposed to the deep definitions.

\subsection{Relations between Classes of Properties}

The basic relations between classical, deep, and shallow definitions, are the following: deep definitions are more general than basic, existential ones, and the shallow definitions are more general than the deep ones, in a sense that is explained formally in the following.

4.1.1 Deep definitions vs. classical definitions. We first note that, in the particular case where the quantifiers are all existential, the deep definitions of the properties (Definition 6) correspond to the classical CSP notions, simply because we have out $=$ sol in that case; in other words our definitions truly are generalizations of the classical definitions. In the general case, when the quantifiers are not restricted to be existential, we can still ignore the quantifier prefix and apply the classical definitions to the resulting existentially quantified CSP. The relations between the original QCSP and the relaxed CSP are the following:

(1) The deductions made using the classical definitions are correct: a property detected on the existentially quantified CSP, using the classical definitions, will also hold for the QCSP.

(2) This reasoning is incomplete: if we do not take into account the quantifier prefix as our new definitions do, some properties cannot be detected.

The correctness can be stated formally as follows:

Proposition 3. Let $\phi=\langle X, Q, D, C\rangle$ be a $Q C S P$ and let $\psi$ be the same $Q C S P$ but in which all quantifiers are existential, i.e., $\psi=\left\langle X, Q^{\prime}, D, C\right\rangle$, with $Q_{x}^{\prime}=\exists$, for all $x \in X$. We have (forall $x_{i}, a, b, V$ ):

- inconsistent $^{\psi}\left(x_{i}, a\right) \rightarrow$ inconsistent $^{\phi}\left(x_{i}, a\right)$;

$-d$-fixable ${ }^{\psi}\left(x_{i}, a\right) \rightarrow d$-fixable ${ }^{\phi}\left(x_{i}, a\right) ;$

-d-substitutable $\left(x_{i}, a, b\right) \rightarrow d$-substitutable ${ }^{\phi}\left(x_{i}, a, b\right)$;

-d-removable ${ }^{\psi}\left(x_{i}, a\right) \rightarrow$ d-removable ${ }^{\phi}\left(x_{i}, a\right)$;

$-d$-interchangeable ${ }^{\psi}\left(x_{i}, a, b\right) \rightarrow d$-interchangeable ${ }^{\phi}\left(x_{i}, a, b\right)$;

$\operatorname{determined}^{\psi}\left(x_{i}\right) \rightarrow$ determined $^{\phi}\left(x_{i}\right)$;

$-d$-irrelevant ${ }^{\psi}\left(x_{i}\right) \rightarrow$ d-irrelevant ${ }^{\phi}\left(x_{i}\right)$;

$-\operatorname{dependent}^{\psi}\left(V, x_{i}\right) \rightarrow \operatorname{dependent}^{\phi}\left(V, x_{i}\right)$. 
We note that the idea of relaxing universal quantifiers and approximating a QCSP by a classical, existential CSP, has been considered implicitly by several authors: the solver presented in [Benedetti et al. 2007] is built on top of a classical CP solver and its propagation mechanism essentially relies on the classical notion of inconsistency; other authors [Mamoulis and Stergiou 2004; Gent et al. 2005] have investigated the use of substitutability in QCSP; here again the notion they have used was essentially the classical, existential one.

Replacing a universal quantifier by an existential one is but one way to obtain a relaxation of a QCSP. In [Ferguson and O'Sullivan 2007], a more comprehensive list of relaxation techniques is studied. Interestingly this work essentially defines a relaxation as a transformation that guarantees that if the relaxation is false, then so is the original problem. In other words, the notion of relaxation is based on the truth of the QCSP. Proposition 3 shows that quantifier relaxation provides a way to do approximate reasoning on other properties than truth.

The incompleteness of the reasoning on the existential relaxation is easily seen on an example:

Example 2. (Ctd.) Consider the QCSP:

$$
\exists x_{1} \in[2,3] \forall x_{2} \in[3,4] \exists x_{3} \in[3,6] . x_{1}+x_{2} \leq x_{3}
$$

(See Fig. 1.) Noticeable properties are: inconsistent $\left(x_{1}, 3\right), \operatorname{implied}\left(x_{1}, 2\right)$, $d$-fixable $\left(x_{1}, 2\right), d$-removable $\left(x_{1}, 3\right), d$-substitutable $\left(x_{1}, 3,2\right)$, determined $\left(x_{1}\right)$.

On the contrary if we apply the classical definition or, equivalently, consider the $C S P \exists x_{1} \in[2,3] \exists x_{2} \in[3,4] \exists x_{3} \in[3,6] . x_{1}+x_{2} \leq x_{3}$, none of the properties holds, because of the tuple $\langle 3,3,6\rangle$.

This confirms that the properties we have defined are new notions which do make a difference compared to classical CSP notions, and which allow a finer reasoning taking into account the quantifier prefix as well as the constraints themselves.

4.1.2 Shallow properties vs. deep properties. To complete the picture, we have the following relations between deep and shallow notions (the deep ones are more restrictive):

Proposition 4. For all variables $x_{i}$ and values $a$ and $b$, we have:

$$
\begin{aligned}
& \text {-d-fixable }\left(x_{i}, a\right) \rightarrow \text { s-fixable }\left(x_{i}, a\right) ; \\
& \text {-d-removable }\left(x_{i}, a\right) \rightarrow \text { s-removable }\left(x_{i}, a\right) ; \\
& \text { - } d \text {-substitutable }\left(x_{i}, a, b\right) \rightarrow \text { s-substitutable }\left(x_{i}, a, b\right) ; \\
& \text {-d-interchangeable }\left(x_{i}, a, b\right) \rightarrow \text { s-interchangeable }\left(x_{i}, a, b\right) ; \\
& \text {-d-irrelevant }\left(x_{i}\right) \rightarrow \text { s-irrelevant }\left(x_{i}\right) .
\end{aligned}
$$

Note that whether a property holds is always dependent on the quantification order. In the case of shallow definitions, this is even more true, because the ordering matters even within a block of variables of the same nature, for instance when the quantifiers are all existential. To see that, consider the QCSP:

$$
\exists x_{1} \in[1,2] \exists x_{2} \in[3,4] \exists x_{3} \in[4,6] . x_{1}+x_{2}=x_{3} .
$$

ACM Transactions on Computational Logic, Vol. V, No. N, October 2018. 
Value 1 is shallow-substitutable to 2 for $x_{1}$, and $x_{1}$ is shallow-irrelevant, while 1 is not deep-substitutable to 2 for $x_{1}$ (i.e., substitutable in the classical sense), nor is $x_{1}$ deep-irrelevant. The intuition behind this is that here we consider that $x_{1}$ is assigned first, and at this step the two choices are equivalent. In other words, the property holds because we are considering the ordering $x_{1}, x_{2}, x_{3}$.

Interestingly, shallow properties, and shallow substitutability in particular, provide a new, general form of properties even for the case of classical CSP. These properties are more general because they take into account information on a particular variable ordering. An interesting question is to determine the variable ordering that allows to detect the highest number of substitutability properties in a given CSP.

\subsection{Relations between Properties}

As in the classical case [Bordeaux et al. 2004], we also have relations between the properties, for instance a value that is implied is also deep-fixable (and therefore also shallow fixable); a variable that is (deep/shallow) irrelevant is also (deep/shallow) fixable to any value, etc. We list the most remarkable of these relations in the next proposition:

Proposition 5. The following relations hold between the properties (forall $x_{i}$, $a$ and $b)$ :

-inconsistent $\left(x_{i}, a\right) \rightarrow \forall b \in D_{x_{i}} . d$-substitutable $\left(x_{i}, a, b\right)$;

-implied $\left(x_{i}, a\right) \leftrightarrow \forall b \in D_{x_{i}} \backslash\{a\}$. inconsistent $\left(x_{i}, b\right)$;

-implied $\left(x_{i}, a\right) \rightarrow d$-fixable $\left(x_{i}, a\right)$;

-inconsistent $\left(x_{i}, a\right) \rightarrow$ d-removable $\left(x_{i}, a\right)$;

$-\exists b \in D_{x_{i}} \backslash\{a\}$. d-substitutable $\left(x_{i}, a, b\right) \rightarrow$ d-removable $\left(x_{i}, a\right)$;

$\exists b \in D_{x_{i}} \backslash\{a\}$. s-substitutable $\left(x_{i}, a, b\right) \rightarrow$ s-removable $\left(x_{i}, a\right)$;

-d-fixable $\left(x_{i}, b\right) \leftrightarrow \forall a \in D_{x_{i}} . d$-substitutable $\left(x_{i}, a, b\right)$;

${ }_{-}$-fixable $\left(x_{i}, b\right) \leftrightarrow \forall a \in D_{x_{i}}$. s-substitutable $\left(x_{i}, a, b\right)$;

-d-irrelevant $\left(x_{i}\right) \leftrightarrow \forall a \in D_{x_{i}} . d$-fixable $\left(x_{i}, a\right)$;

${ }_{\text {- s-irrelevant }}\left(x_{i}\right) \leftrightarrow \forall a \in D_{x_{i}}$. s-fixable $\left(x_{i}, a\right)$.

\section{SIMPLIFICATIONS ALLOWED WHEN THE PROPERTIES HOLD}

The goal of reasoning on the properties of a QCSP is typically to simplify the problem. In the cases we are interested in, this can be done in two ways: (1) by removing an element from the list of values to consider for one of the variables, or (2) by instantiating a variable to a particular value. Such simplifications are helpful for backtrack search algorithms, which are typically considered when solving QCSP.

We now show that the properties we defined allow simplifications that are correct, in the sense that they do not alter the truth of the QCSP:

- If a value is removable for a given variable, then removing the value from the domain of that variable does not change the truth of the problem.

- If a value is fixable to a particular value for a given variable, then instantiating the variable to this value does not change the truth of the problem. 
The interest of the other properties lies essentially in their relation with the two fundamental properties of removability and fixability, as expressed by Prop. 5. For instance, an implied value is of interest essentially because it is fixable, and an irrelevant variable is of interest essentially because it is fixable to any value of its domain. Similarly, the interest of, e.g., inconsistent and substitutable values is that they are removable. We therefore focus on proving the correctness of the two notions of removability and fixability, and we will consider their shallow forms: recall that, by Prop. 4, the shallow are the stronger ones; a value which is deep-removable or deep-fixable is also shallow-removable or shallow-fixable, respectively.

\subsection{Simplifying Existental Variables}

Our whole game-theoretic approach is naturally biased towards existential variables: the notion of strategy considers that the values for the universal variables can be arbitrary, and specifies the values that should be taken for the existential ones. As a consequence, the approach is more naturally fitted to make deductions on the existential variables, and we first focus on this case.

The simplifications allowed for an existential variable when the removability property holds rely on the following Proposition:

Proposition 6. Let $\phi=\langle X, Q, D, C\rangle$ be a $Q C S P$ in which value $a \in D_{x_{i}}$ is shallow-removable for an existential variable $x_{i}$, and let $\phi^{\prime}$ denote the same QCSP in which value a is effectively removed (i.e., $\phi^{\prime}=\left\langle X, Q, D^{\prime}, C\right\rangle$ where $D_{x_{i}}^{\prime}=D_{x_{i}} \backslash\{a\}$ and $\left.D_{x_{j}}^{\prime}=D_{x_{j}}, \forall j \neq i\right)$. Then $\phi$ is true iff $\phi^{\prime}$ is true.

The simplifications allowed for an existential variable when the fixability property holds rely on the following Proposition:

Proposition 7. Let $\phi=\langle X, Q, D, C\rangle$ be a QCSP in which value $a \in D_{x_{i}}$ is shallow-fixable for an existential variable $x_{i}$, and let $\phi^{\prime}$ denote the same QCSP in which value a is effectively fixed (i.e., $\phi^{\prime}=\left\langle X, Q, D^{\prime}, C\right\rangle$ where $D_{x_{i}}^{\prime}=\{a\}$ and $\left.D_{x_{j}}^{\prime}=D_{x_{j}}, \forall j \neq i\right)$. Then $\phi$ is true iff $\phi^{\prime}$ is true.

\subsection{Simplifying Universal Variables}

To allow a proper, symmetric treatment of all variables of QCSPs it is necessary to also define how to make deductions on universal variables. The way this can be done has been suggested by several authors in the literature and is developed, for instance, in [Bordeaux and Zhang 2007]: to make deductions on the universal variables, which represent the "moves of the opponent", we have to reason on the negation of the formula, which captures the "winning strategies of the opponent".

We say that a value is dual-shallow-removable if it is shallow-removable in the negation of the considered QCSP, and that it is dual-shallow-fixable if it is shallowfixable in this negation. The simplifications allowed for a universal variable when the removability property holds rely on the following Proposition:

Proposition 8. Let $\phi=\langle X, Q, D, C\rangle$ be a QCSP in which value $a \in D_{x_{i}}$ is dual-shallow-removable for a universal variable $x_{i}$, and let $\phi^{\prime}$ denote the same QCSP in which value a is effectively removed (i.e., $\phi^{\prime}=\left\langle X, Q, D^{\prime}, C\right\rangle$ where $D_{x_{i}}^{\prime}=D_{x_{i}} \backslash$ $\{a\}$ and $\left.D_{x_{j}}^{\prime}=D_{x_{j}}, \forall j \neq i\right)$. Then $\phi$ is true iff $\phi^{\prime}$ is true.

ACM Transactions on Computational Logic, Vol. V, No. N, October 2018. 
The simplifications allowed for a universal variable when the fixability property holds rely on the following Proposition:

Proposition 9. Let $\phi=\langle X, Q, D, C\rangle$ be a QCSP in which value a $\in D_{x_{i}}$ is dual-shallow-fixable for an universal variable $x_{i}$, and let $\phi^{\prime}$ denote the same QCSP in which value a is effectively fixed (i.e., $\phi^{\prime}=\left\langle X, Q, D^{\prime}, C\right\rangle$ where $D_{x_{i}}^{\prime}=\{a\}$ and $\left.D_{x_{j}}^{\prime}=D_{x_{j}}, \forall j \neq i\right)$. Then $\phi$ is true iff $\phi^{\prime}$ is true.

\section{COMPLEXITY RESULTS}

In this section, we study the complexity of the problem of determining whether the properties defined in Definitions 6 and 7 hold. As was to be expected, our results show that the problem is in general intractable, and we essentially obtain PSPACE-completeness results. In other words the complexity of checking one of the properties is typically the same as the complexity of determining whether the QCSP is true [Papadimitriou 1994; Stockmeyer and Meyer 1973].

\subsection{Encoding Issues}

To analyze the complexity, a few words are needed on the encoding of the QCSP $\langle X, Q, D, C\rangle$. Def. 1 did not specify anything on this issue, because the encoding did not have any consequence on the results of previous sections. We assume that $X$ and $Q$ are encoded in the natural way, i.e., as a list. For the set of domains $D$, two choices may be considered: a domain can be encoded as a list of allowed values or as an interval, in which case its two bounds need to be encoded. Our results will hold independently of whether the interval or domain representation is chosen. The main question is how the constraints are defined. Some examples of representation formalisms are the following:

I The domain is Boolean, i.e., $B=\{0,1\}$, and $C$ is defined as a Boolean circuit.

II The domain is Boolean, i.e., $B=\{0,1\}$, and $C$ is put in Conjunctive Normal Form, i.e., it is a conjunction of clauses (disjunctions of literals, each of which is a variable or its negation).

III $C$ is a conjunction of constraints, each of which is represented in extension as a table (e.g., binary) which lists all tuples that are accepted.

IV $C$ is a conjunction of constraints, each of which is represented by a numerical (linear or polynomial) equality or inequality.

$\mathrm{V} C$ is a polynomial-time program (written in any universal language, for instance the Turing machine) which, given a tuple $t$, determines whether $t \in$ sol.

In all cases we impose the restriction that testing whether $t \in$ sol be feasible in polynomial time. The fifth encoding represents the most general possible encoding satisfying this restriction: we shall consider it when we want to check that a result holds for any encoding in which testing whether $t \in$ sol can be done in polynomial time.

Using encoding (V) to capture the notion of "most general encoding" is therefore convenient, but an important point is that the 4 other formalisms are essentially as concise as formalism (V). If the domain is Boolean, then if sol can be represented by a program $P$ (in the sense that $P(t)=1$ iff $t \in$ sol) and if the execution of 
$P$ requires a memory bounded by $S$ and a time bounded by $L$, then the set sol can be also represented by a Boolean circuit of size polynomial in $S, L$, and the length of the text of the program $P$, using the technique used by Cook in proving that SAT is NP-complete. In other words, for Boolean domains, formalism (I) is as expressive as formalism (V). Now the relations between formalism (I) and formalisms (II) to (IV) are well-known: we can reduce a circuit to a CNF involving only clauses of size at most three (3CNF) by introducing existential variables, and it is straightforward to reduce a $3 \mathrm{CNF}$ to formalism (III) or formalism (IV). The complexities of our problems for (I) to (V) will therefore be equivalent except for minor refinements occurring at intermediate levels of the polynomial hierarchy (Prop 13), where introducing existential variables makes a little difference.

\subsection{A Common Upper Bound: PSPACE}

The most difficult side of our complexity characterizations is to prove membership in PSPACE. It is indeed not completely obvious at first that the properties we have studied can be verified in polynomial space. The key point is to notice that a polynomial space algorithm exists to recognize the set of outcomes. Considering representation $(\mathrm{V})$, we have the following:

Proposition 10. Let $\phi=\langle X, Q, D, C\rangle$ be a QCSP. Given a tuple $t \in \prod_{x \in X} D_{x}$, we denote by $B$ the conjunction of constraints:

$$
\bigwedge_{x_{i} \in E}\left(\left(\bigwedge_{y \in A_{i-1}} y=t_{y}\right) \rightarrow\left(x_{i}=t_{x_{i}}\right)\right)
$$

The $Q C S P \psi=\langle X, Q, D, B \cup C\rangle$ is true iff $t \in$ out $^{\phi}$.

Note that $B \cup C$ can be expressed concisely in formalism (V). The conjunction of constraints added in (3) makes sure that any winning strategy of $\psi$ contains $t$ as a scenario.

A direct corollary of Prop. 10 is that checking whether a particular tuple $t$ belongs to the set of outcomes of a QCSP $\phi$ can be done in polynomial space, simply by solving $\psi$. This is true for any representation of the constraints that respects the restriction that testing whether $t \in$ sol be feasible in polynomial time ${ }^{2}$. Now being able to test in polynomial space whether a tuple is an outcome, the membership in PSPACE of all properties becomes clear: for instance if we consider inconsistency $\left(\forall t \in\right.$ out. $t_{x_{i}} \neq a$ ) we can enumerate all tuples in lexicographical order, determine whether each of them is an outcome, and whether it satisfies the implication $t \in$ out. $t_{x_{i}} \neq a$. The precise list of results will be given in the next section, where we state completeness results (including both hardness and membership for the considered class).

Example 4. Let us illustrate the idea of Prop. 10 on a simple example. Consider the QCSP $\exists x_{1} . \forall y_{1} . \exists x_{2} . \forall y_{2} . \exists x_{3} . C$, where the domain of each variable is, for instance $\{0,1\}$. We want to determine whether the tuple $\left\langle x_{1}=0, y_{1}=0, x_{2}=\right.$

${ }^{2}$ In fact this condition could itself be considerably relaxed: the PSPACE membership result holds under the very general condition that testing whether $t \in$ sol be feasible in polynomial space.

ACM Transactions on Computational Logic, Vol. V, No. N, October 2018. 
$\left.0, y_{2}=0, x_{3}=0\right\rangle$ is an outcome of the QCSP. This can be done by solving the QCSP in which the constraints of (3) are added:

$\exists x_{1} \cdot \forall y_{1} \cdot \exists x_{2} . \forall y_{2} \cdot \exists x_{3} . C \wedge\left(x_{1}=0 \wedge\left(y_{1}=0 \rightarrow x_{2}=0\right) \wedge\left(\left(y_{1}=0 \wedge y_{2}=0\right) \rightarrow x_{3}=0\right)\right)$.

It might be useful to mention a possible source of confusion: it is the case that our PSPACE membership results hold for formalism (4), since it respects our restriction. This is true even if the domains $D_{x}$ are represented by intervals: even though an interval whose bounds are $n$-bit integers represents in general a set of values of cardinality exponential in $n$, we can always iterate on these values using polynomial space. This should be contrasted with classical complexity results related to arithmetics: in general deciding the truth of quantified linear constraints is extremely complex (hard for $\operatorname{NDTIME}\left(2^{2^{n}}\right)$ by the Fischer-Rabin theorem [Fischer and Rabin 1974], and therefore provably not in PSPACE $\subseteq$ EXPTIME), and if we consider quantified polynomial constraints the problem becomes undecidable (Gödel's theorem). The key point is that in these cases the values of the variables can grow extremely large; as long as we bound the domains explicitly this problem does not arise, which is why we remain within PSPACE.

\subsection{Complexity Characterizations}

We now list the complexity results we obtain. These results hold for any of the 5 representations we have mentioned.

Proposition 11. Given a $Q C S P \phi=\langle X, Q, D, C\rangle$, the problems of deciding whether:

-value $a \in D_{x_{i}}$ is d-fixable, $d$-removable, inconsistent, implied for variable $x_{i} \in$ $X$,

—value $a \in D_{x_{i}}$ is d-substitutable to or d-interchangeable with $b \in D_{x_{i}}$ for variable $x_{i} \in X$,

-variable $x_{i} \in X$ is dependent on variables $V \subseteq X$, or is d-irrelevant

are PSPACE-complete.

An analogous result holds for the shallow properties:

Proposition 12. Given a QCSP $\phi=\langle X, Q, D, C\rangle$, the problems of deciding whether:

-value $a \in D_{x_{i}}$ is s-fixable, s-removable for variable $x_{i} \in X$,

-value $a \in D_{x_{i}}$ is s-substitutable to or s-interchangeable with $b \in D_{x_{i}}$ for variable $x_{i} \in X$,

-variable $x_{i} \in X$ is s-irrelevant

are PSPACE-complete.

As usual when considering quantified constraints, the complexity increases with the number of quantifier alternations, more precisely each additional alternation brings us one level higher in the Polynomial Hierarchy [Stockmeyer 1976]. The precise level that is reached is dependent on the considered property and on many details, including the formalism used for the encoding of the QCSP. We shall not 
list all results but instead we characterize, as an example, the complexity obtained in a particular setting, i.e., for the "deep" definitions of the properties, in the case where the QCSP starts with an existential quantifiers, and where its constraints are encoded as a Boolean circuit.

We call $\Sigma_{k}$ QCSPs the QCSPs with at most $k$ quantifier alternations and whose first variables are existential. We have the following results:

Proposition 13. Given a $\Sigma_{k} Q C S P \phi=\langle X, Q, D, C\rangle$ encoded using Formalism (I), the problems of deciding whether:

-value $a \in D_{x_{i}}$ is deep-fixable, deep-removable, inconsistent, implied for variable $x_{i} \in X$,

- value $a \in D_{x_{i}}$ is deep-substitutable to or deep-interchangeable with $b \in D_{x_{i}}$ for variable $x_{i} \in X$,

-variable $x_{i} \in X$ is dependent on variables $V \subseteq X$, or is deep-irrelevant,

are $\Pi_{k}^{p}$-hard and belong to $\Pi_{k+1}^{p}$. Moreover, for deep inconsistency, implication, determinacy and dependence, the problems are more precisely $\Pi_{k}^{p}$-complete.

In particular, it was reported in [Bordeaux et al. 2004] that these problems are coNP-complete for purely existential QCSPs.

Why the precise results are less regular than in previous cases is because the precise number of quantifier alternations is impacted by many factors. For instance, if we consider a Quantified Boolean Formula $\exists X . \forall Y . F(X, Y)$, where $X$ and $Y$ are vectors of Boolean variables and $F$ is a Boolean circuit, then putting $F$ into CNF will produce a formula of the form $\exists X . \forall Y . \exists Z . G(X, Y, Z)$, and this sometimes incurs a difference of one level in the polynomial hierarchy between Formalism (I) and Formalisms (II) to (IV). Similarly, there is a difference between shallow and deep properties in that shallow properties are themselves usually stated with more quantifier alternations, a typical form being "forall outcomes, there exists an outcome". What is obviously true for all properties in any case, however, is if we consider QCSPs with a limited number of quantifier alternations, the level reached in the polynomial hierarchy is also bounded.

\section{LOCAL REASONING}

The previous section shows that all of the properties we are interested in are computationally difficult to detect - in fact as difficult as the resolution of the QCSP problem itself. There are nonetheless particular cases where a property can be cheaply revealed. In CSP solvers the most widely used way of detecting properties cheaply is by using local reasoning: instead of analysing the whole problem at once, thereby facing its full complexity, we analyse it bit by bit (typically constraint by constraint). Depending on the property we know how deductions made on the bits generalize to the whole QCSP. For instance:

- In the case of inconsistency, a deduction made on one single constraint generalizes to the whole CSP. For instance, if we have a CSP $\exists x \in[0,5] . y \in[0,5] . x>y \wedge C$, we can deduce from the constraint $x>y$ that value 0 is inconsistent for $x$, without having to worry of which other constraints are present in $C$.

ACM Transactions on Computational Logic, Vol. V, No. N, October 2018. 
- In the case of substitutability, a deduction is valid for the whole QCSP if it can be checked independently for each and every constraint. For instance if we have the CSP $\exists x \in[0,5] . y \in[0,5] . x>1 \wedge x \leq y$, we can deduce that value value 3 is substitutable to 2 for $x$. This is the case because the substitutability property holds for both constraints $x>1$ and $x \leq y$. If, however, there were a third constraint, we would have to make sure that the property holds for it as well before deducing that it holds for the whole CSP. The situation is slightly less advantageous than for inconsistency because we have to consider each constraint before making a deduction, but it is nevertheless of interest - analysing the constraints one by one is typically much cheaper than analysing the whole CSP at once.

Following the classical CSP approach, we investigate the use of local reasoning as a means to cheaply detect the properties we have proposed.

\subsection{Positive Results}

Our first result is that using local reasoning allows to detect the deep properties except removability. Depending on the property one of the two forms of generalization mentioned before is correct.

Proposition 14. Let $\phi=\langle X, Q, D, C\rangle$ be a QCSP where $C=\left\{c_{1}, \ldots, c_{m}\right\}$. We denote by $\phi_{k}$ the $Q C S P\left\langle X, Q, D,\left\{c_{k}\right\}\right\rangle$ in which only the $k$-th constraint is considered. We have, for all $x_{i} \in X, V \subseteq X$, and $a, b \in D_{x_{i}}$ :

$-\left(\bigvee_{k \in 1 . . m}\right.$ inconsistent $\left.^{\phi_{k}}\left(x_{i}, a\right)\right) \rightarrow$ inconsistent $^{\phi}\left(x_{i}, a\right)$;

$-\left(\bigvee_{k \in 1 . . m} \operatorname{implied}^{\phi_{k}}\left(x_{i}, a\right)\right) \rightarrow \operatorname{implied}^{\phi}\left(x_{i}, a\right) ;$

$-\left(\bigwedge_{k \in 1 . . m} d\right.$-fixable $\left.{ }^{\phi_{k}}\left(x_{i}, a\right)\right) \rightarrow$ d-fixable ${ }^{\phi}\left(x_{i}, a\right) ;$

$-\left(\bigwedge_{k \in 1 . . m} d\right.$-substitutable $\left.\phi^{\phi_{k}}\left(x_{i}, a, b\right)\right) \rightarrow d$-substitutable ${ }^{\phi}\left(x_{i}, a, b\right) ;$

$-\left(\bigwedge_{k \in 1 . . m}\right.$ d-interchangeable $\left.{ }^{\phi_{k}}\left(x_{i}, a, b\right)\right) \rightarrow$ d-interchangeable ${ }^{\phi}\left(x_{i}, a, b\right)$;

$-\left(\bigvee_{k \in 1 . . m}\right.$ determined $\left.^{\phi_{k}}\left(x_{i}\right)\right) \rightarrow$ determined $^{\phi}\left(x_{i}\right)$;

$-\left(\bigwedge_{k \in 1 . . m}\right.$ d-irrelevant $\left.{ }^{\phi_{k}}\left(x_{i}\right)\right) \rightarrow$ d-irrelevant ${ }^{\phi}\left(x_{i}\right)$;

$-\left(\bigvee_{k \in 1 . . m}\right.$ dependent $\left.^{\phi_{k}}\left(V, x_{i}\right)\right) \rightarrow \operatorname{dependent}^{\phi}\left(V, x_{i}\right)$.

\subsection{Negative Results}

It was noticed in [Bordeaux et al. 2004] that, even in the non-quantified case, deep removability is not as well-behaved as the other deep properties since it is not possible to detect it using local reasoning. This was seen on an example, which we borrow from this paper:

Example 5. Consider the CSP

$$
\exists x \in\{1,2,3\} . \exists y \in\{1,2,3\} .(x \leq y, y \leq x, x \neq 1, x \neq 3)
$$

If we consider each of the four constraints, then we find that value 2 is removable for $x$. But obviously value 2 is not removable for the CSP as the only solution is indeed $x=2, y=2$. 
A similar problem occurs when we consider the shallow definitions: it is incorrect, in general, to use local reasoning to detect these versions of the properties ${ }^{3}$. Here again this can be seen on a simple example:

EXAmple 6. Consider the (Q)CSP

$$
\exists x_{1} \in\{0,1\} . \exists x_{2} \in\{0,1\} .\left(x_{1}=x_{2} \wedge x_{2}=1\right)
$$

It is the case that variable $x_{1}$ is shallow-fixable to value 0 w.r.t. constraint $x_{1}=x_{2}$; and variable $x_{1}$ is also shallow-fixable to value 0 w.r.t. constraint $x_{2}=1$. Despite of that, $x_{1}$ is not shallow-fixable to 0 in the QCSP, as there is simply no solution with $x_{1}=0$.

The shallow definitions therefore have to be considered carefully: they are more general than the deep properties, but they have to be detected by other means than local reasoning. This is somewhat reminiscent of what happens with the removability property, whose generality comes at the price of being a less wellbehaved property than substitutability or inconsistency.

\section{CONCLUDING REMARKS}

\subsection{Related Works}

A number of works related to Quantified CSP have considered particular cases of the properties we have attempted to study systematically in this paper. Most of these works have been mentioned throughout the paper, notably [Mamoulis and Stergiou 2004] for their use of substitutability; we also note the work done by Peter Nightingale in his thesis, which devotes large parts to the consistency property [Nightingale 2005]. The notions considered in these works are related to our proposals but typically less general, because our definitions finely take into account the quantifiers. For substitutability for instance, the definition used in [Mamoulis and Stergiou 2004] was essentially the classical (existential) definition. For consistency, our definition subsumes the notions proposed by [Bordeaux and Monfroy 2002] or [Nightingale 2005]. Our general definition nevertheless leaves open the question of how to efficiently detect inconsistent values, and these proposals can be seen as particular ways of using local reasoning to detect inconsistent values. This situation is quite closely related to works in CSP, where many notions of local consistency can be defined. These notions have different merits that can be evaluated experimentally, but they all share the basic property of being ways to detect (globally) inconsistent values, which explains why they are correct.

We also note that more advanced studies are available for the particular case of Boolean quantified constraints. In these works some techniques have been proposed that specifically take into account the quantifier prefix. However, contrary to ours, these proposals are restricted to Boolean domains. For instance in [Rintanen 1999; Cadoli et al. 2002], several techniques are proposed to fix and remove values. These works have shown that detecting properties is essential and can lead to a consistent pruning of the search space, but no clear and general framework to understand these properties was available.

\footnotetext{
${ }^{3}$ This corrects an error in [Bordeaux et al. 2005], where we wrongly stated that local reasoning is valid for all properties.
}

ACM Transactions on Computational Logic, Vol. V, No. N, October 2018. 
An interesting, recent related work is [Audemard et al. 2007], which initiates the study of symmetries in Quantified Boolean Formulae. Symmetries are related to the notion of interchangeability but are in a sense a more general concept. Our feeling is that the idea of using the notion of outcome to define constraint properties may be applicable to this class of properties as well. Symmetries are a complex and fascinating topic; an interesting perspective for future work will be to see if our framework can help understanding them in the general context of quantified CSP.

\subsection{Conclusion}

A primary goal of our work was to state the definitions in a way that is formal and amenable to proofs. In previous QCSP literature, it is fair to say that formal proofs were scarce, probably because facts that are trivial to prove in CSP tend to become complex to write formally when quantifiers come into play. Quantifiers can be complex to reason with, and it is sometimes easy to make wrong assumptions on some properties, as we saw ourselves when finding the error we made in the preliminary version of this paper (Section 6). Because of this difficulty, we wanted in this work to build solid foundations on which the deductions made in QCSP solvers can rely.

\section{REFERENCES}

Audemard, G., Jabbour, S., And Saïs, L. 2007. Symmetry breaking in Quantified Boolean Formulae. In Proc. of Int. Joint. Conf. on Artificial Intelligence (IJCAI). Morgan Kaufmann, 2262-2267.

Benedetti, M. 2004. Evaluating QBF via symbolic skolemization. In Proc. of Int. Conf. on Logic for Programming, Artificial Intelligence and Reasoning (LPAR). Springer, 285-300.

Benedetti, M., Lallouet, A., And Vautard, J. 2007. QCSP made practical by virtue of restricted quantification. In Proc. of Int. Joint. Conf. on Artificial Intelligence (IJCAI). Morgan Kaufmann, 38-43.

Bordeaux, L., Cadoli, M., And Mancini, T. 2004. Exploiting fixable, removable and determined values in constraint satisfaction problems. In Proc. of Int. Conf. on Logic for Programming, Artificial Intelligence and Reasoning (LPAR). Springer, 270-284.

Bordeaux, L., Cadoli, M., And Mancini, T. 2005. CSP properties for quantified constraints: Definitions and complexity. In Proc. of Amer. Conf. on Artificial Intelligence (AAAI). AAAI Press, 360-365.

Bordeaux, L. and Monfroy, E. 2002. Beyond NP: Arc-consistency for quantified constraints. In Proc. of Int. Conf. on Principles and Practice of Constraint Programming (CP). Springer, 371-386.

Bordeaux, L. And Zhang, L. 2007. A solver for quantified Boolean and linear constraints. In Proc. of Int. Symp. on Applied Computing (SAC). ACM, To appear.

Börner, F., Bulatov, A., Jeavons, P., And Krokhin, A. 2003. Quantified constraints: Algorithms and complexity. In Proc. of Int. Conf. on Computer Science Logic (CSL). Springer, $58-70$.

Buening, H. K., Karpinski, M., And Flogel, A. 1995. Resolution for Quantified Boolean Formulas. Information and Computation 117, 1, 12-18.

Cadoli, M., Giovanardi, A., And Schaerf, M. 1999. An algorithm to evaluate quantified boolean formulae. In Proc. of Amer. Conf. on Artificial Intelligence (AAAI). AAAI/MIT Press, 262-267.

Cadoli, M., Schaerf, M., Giovanardi, A., And Giovanardi, M. 2002. An algorithm to evaluate Quantified Boolean Formulae and its experimental evaluation. J. of Automated Reasoning 28, 2, 101-142. 
Chen, H. 2004a. Collapsibility and consistency in quantified constraint satisfaction. In Proc. of Amer. Conf. on Artificial Intelligence (AAAI). AAAI Press, 155-160.

Chen, H. 2004b. Quantified constraint satisfaction and bounded treewidth. In Proc. of Euro. Conf. on Artificial Intelligence (ECAI). IOS Press, 161-165.

Ferguson, A. and O'Sullivan, B. 2007. Quantified Constraint Satisfaction Problems: from relaxations to explanations. In Proc. of Int. Joint. Conf. on Artificial Intelligence (IJCAI). Morgan Kaufmann, 74-79.

Fischer, M. J. And Rabin, M. O. 1974. Super-exponential complexity of Presburger Arithmetics. In Complexity of Computation, R. Karp, Ed. 27-41.

Freuder, E. C. 1991. Eliminating interchangeable values in constraint satisfaction problems. In Proc. of Amer. Conf. on Artificial Intelligence (AAAI). AAAI Press, 227-233.

Gent, I., Nightingale, P., And Stergiou, K. 2005. QCSP-Solve: A solver for quantified constraint satisfaction problems. In Proc. of Int. Joint. Conf. on Artificial Intelligence (IJCAI). Morgan Kaufmann, 138-143.

Gent, I. P., Nightingale, P., And Rowley, A. 2004. Encoding quantified CSPs as Quantified Boolean Formulae. In Proc. of Euro. Conf. on Artificial Intelligence (ECAI). IOS Press, 176180.

Mackworth, A. 1977. Consistency in networks of relations. Artificial Intelligence 8, 99-118.

Mamoulis, N. And Stergiou, K. 2004. Algorithms for Quantified Constraint Satisfaction Problems. Tech. Rep. APES-79-2004, Apes research group.

Monasson, R., Zecchina, R., Kirkpatrick, S., Selman, B., and Troyansky, L. 1999. Determining computational complexity from characteristic 'phase transitions'. Nature 400, 133-137.

Nightingale, P. 2005. Consistency for quantified constraint satisfaction problems. In Proc. of Int. Conf. on Principles and Practice of Constraint Programming (CP). Springer, 792-796.

Papadimitriou, C. H. 1994. Computational Complexity. Addison Wesley.

Rintanen, J. 1999. Improvements to the Evaluation of Quantified Boolean formulae. In Proc. of Int. Joint. Conf. on Artificial Intelligence (IJCAI). Morgan Kaufmann, 1192-1197.

Samulowitz, H. And Bacchus, F. 2006. Binary clause reasoning in QBF. In Proc. of Int. Conf. on Theory and Applications of Satisfiability Testing (SAT). Springer, 353-367.

Samulowitz, H., Davies, J., And Bacchus, F. 2006. Preprocessing QBF. In Proc. of Int. Conf. on Principles and Practice of Constraint Programming (CP). Springer, 514-529.

Stockmeyer, L. J. 1976. The polynomial-time hierarchy. Theoretical Computer Science (TCS) 3, 1, 1-22.

Stockmeyer, L. J. And Meyer, A. R. 1973. Word problems requiring exponential time: Preliminary report. In Proc. of Symp. on Theory of Computing (STOC). ACM, 1-9.

Verger, G. And Bessière, C. 2006. A bottom-up approach for solving quantified CSPs. In Proc. of Int. Conf. on Principles and Practice of Constraint Programming (CP). Springer, 635-649.

Zhang, L. 2006. Solving QBF by combining conjunctive and disjunctive normal forms. In Proc. of Amer. Conf. on Artificial Intelligence (AAAI).

Received XXXXX; accepted XXXXX 
THIS DOCUMENT IS THE ONLINE-ONLY APPENDIX TO:

\section{Generalizing Consistency and other Constraint Properties to Quantified Constraints}

\section{LUCAS BORDEAUX}

Microsoft Research

MARCO CADOLI

Università di Roma "La Sapienza"

TONI MANCINI

Università di Roma "La Sapienza"

ACM Transactions on Computational Logic, Vol. V, No. N, October 2018, Pages 1-22.

\section{A. PROOFS OF THE MAIN PROPOSITIONS}

Proposition 1. A QCSP is true (as defined in Section 2.1.2) iff it has a winning strategy.

Proof. Instead of proving this result from scratch we sketch its connection to classical logical results and simply note that the functions used in the definition of the notion of strategy are essentially Skolem functions: it is well-known that, starting from a formula $\forall x_{1} \ldots x_{n} . \exists y . F\left(x_{1}, \ldots x_{n}, y\right)$ with an existentially quantified variable $y$, we can replace $y$ by a function and obtain a second-order formula that is equivalent: $\exists f . \forall x_{1} \ldots x_{n} . F\left(x_{1}, \ldots x_{n}, f\left(x_{1} \ldots x_{n}\right)\right)$.

If the domain $\mathbb{D}$ is additionally fixed and each quantifier is bounded, i.e., if we have a formula of the form: $\forall x_{1} \in D_{x_{1}} \ldots \forall x_{n} \in D_{x_{n}} . \exists y \in D_{y} . F\left(x_{1}, \ldots x_{n}, y\right)$, then the formula is equivalent to:

$$
\exists f . \forall x_{1} \in D_{x_{1}} \ldots \forall x_{n} \in D_{x_{n}} .\left(f\left(x_{1} \ldots x_{n}\right) \in D_{y} \wedge F\left(x_{1}, \ldots x_{n}, f\left(x_{1} \ldots x_{n}\right)\right)\right)
$$

and any interpretation $I$ verifying:

$\langle\mathbb{D}, I\rangle \models \forall x_{1} \in D_{x_{1}} \ldots \forall x_{n} \in D_{x_{n}} .\left(f\left(x_{1} \ldots x_{n}\right) \in D_{y} \wedge F\left(x_{1}, \ldots x_{n}, f\left(x_{1} \ldots x_{n}\right)\right)\right)$

is such that the function $I(f)$ is of signature $\left(\prod_{x_{i} \in\left\{x_{1} \ldots x_{n}\right\}} D_{x_{i}}\right) \rightarrow D_{y}$.

Now given a QCSP, let $F$ be its logical representation as defined in Section 2.1.2, and let $F^{\prime}$ be the Skolem normal form of $F$, obtained by iteratively applying the process described above, for all existential variables. The strategies of the QCSP are exactly the possible interpretations of the Skolem functions of $F^{\prime}$. Furthermore, a strategy is winning (all outcomes are true) iff the first-order (universally quantified)

Permission to make digital/hard copy of all or part of this material without fee for personal or classroom use provided that the copies are not made or distributed for profit or commercial advantage, the ACM copyright/server notice, the title of the publication, and its date appear, and notice is given that copying is by permission of the ACM, Inc. To copy otherwise, to republish, to post on servers, or to redistribute to lists requires prior specific permission and/or a fee.

(C) 2018 ACM 1529-3785/18/1000-0001 $\$ 5.00$ 
part of the formula is true. Consequently a winning strategy exists for the QCSP iff the model-checking problem $\langle\mathbb{D}, I\rangle \models F^{\prime}$ is true, i.e., iff the QCSP is true.

Proposition 2. Deep fixability could equivalently be defined by the condition $\forall t \in$ out.t $\left[x_{i}:=a\right] \in$ sol; Deep substitutability could be equivalently defined by $\forall t \in$ out. $\left(t_{x_{i}}=a\right) \rightarrow\left(t\left[x_{i}:=b\right] \in\right.$ sol $)$; deep removability by $\forall t \in$ out. $\left(t_{x_{i}}=a\right) \rightarrow$ $\left(\exists b \neq a . t\left[x_{i}:=b\right] \in\right.$ sol $)$; and deep irrelevance by $\forall t \in$ out. $\forall b \in D_{x_{i}} . t\left[x_{i}:=b\right] \in$ sol.

ProOF. We consider fixability and we prove that $\forall t \in$ out. $t\left[x_{i}:=a\right] \in$ out holds iff $\forall t \in$ out. $t\left[x_{i}:=a\right] \in \underline{\text { sol }}$ does. The $\rightarrow$ implication is straightforward (out $\subseteq$ sol); we prove the $\leftarrow$ implication. In the case where the QCSP is false (no winning strategy) the implication trivially holds, since out is then empty. Let us therefore prove it in the case where the QCSP is true.

We assume that $\forall t \in$ out. $t\left[x_{i}:=a\right] \in$ sol. Let $t \in$ out; it is clear that the tuple $t\left[x_{i}:=a\right]$ belongs to sol; we have to prove that $t\left[x_{i}:=a\right]$ also belongs to out. For that purpose, we exhibit a winning strategy $s$ such that $t\left[x_{i}:=a\right] \in \operatorname{sce}(s)$.

Let $s^{\prime}$ be a winning strategy such that $t \in \operatorname{sce}\left(s^{\prime}\right)$. Such a strategy exists since $t$ is an outcome. The strategy $s$ will be obtained by modifying $s^{\prime}$ so that all its outcomes assign value $a$ to variable $x_{i}$. More formally, the functions $s_{x_{j}}$ are defined, for each $x_{j} \in E$, as follows:

-If $j=i$ then $s_{x_{j}}(\tau) \doteq a$, for each tuple $\tau \in \prod_{y \in A_{j-1}} D_{y}$;

-Otherwise $s_{x_{j}}$ is simply defined as the function $s_{x_{j}}^{\prime}$.

One can now verify that $\operatorname{sce}(s)=\left\{\tau\left[x_{i}:=a\right]: \tau \in \operatorname{sce}\left(s^{\prime}\right)\right\}$. Two consequences are $t\left[x_{i}:=a\right] \in \operatorname{sce}(s)$, and $\operatorname{sce}(s) \subseteq$ sol, which show that $s$ is a winning strategy such that $t\left[x_{i}:=a\right] \in \operatorname{sce}(s)$.

Similarly, for substitutability we can exhibit a strategy $s$ in which every $t \in \operatorname{sce}\left(s^{\prime}\right)$ such that $t_{x_{i}}=a$ is changed into the scenario $t\left[x_{i}:=b\right]$.

For removability it is convenient to restate the property: removability holds if there exists a function $f$ that associates to every $X$-tuple $t$ a value $f(t) \neq a$, and such that $\forall t \in$ out. $\left(t_{x_{i}}=a\right) \rightarrow\left(t\left[x_{i}:=f(t)\right] \in\right.$ out $)$. We can exhibit a strategy $s$ in which every $t \in \operatorname{sce}(s)$ such that $t_{x_{i}}=a$ is changed into the scenario $t\left[x_{i}:=f(t)\right]$.

For irrelevance we can use the fact that a variable is irrelevant iff it can be fixed to any value of its domain (Prop. 5).

Proposition 3. Let $\phi=\langle X, Q, D, C\rangle$ be a QCSP and let $\psi$ be the same QCSP but in which all quantifiers are existential, i.e., $\psi=\left\langle X, Q^{\prime}, D, C\right\rangle$, with $Q_{x}^{\prime}=\exists$, for all $x \in X$. We have (forall $\left.x_{i}, a, b, V\right)$ :

- inconsistent $^{\psi}\left(x_{i}, a\right) \rightarrow$ inconsistent $^{\phi}\left(x_{i}, a\right)$;

$-d$-fixable ${ }^{\psi}\left(x_{i}, a\right) \rightarrow$ d-fixable ${ }^{\phi}\left(x_{i}, a\right) ;$

-d-substitutable $\left(x_{i}, a, b\right) \rightarrow d$-substitutable ${ }^{\phi}\left(x_{i}, a, b\right)$;

-d-removable ${ }^{\psi}\left(x_{i}, a\right) \rightarrow$ d-removable ${ }^{\phi}\left(x_{i}, a\right)$;

$-d$-interchangeable ${ }^{\psi}\left(x_{i}, a, b\right) \rightarrow d$-interchangeable ${ }^{\phi}\left(x_{i}, a, b\right)$;

$\operatorname{determined}^{\psi}\left(x_{i}\right) \rightarrow$ determined $^{\phi}\left(x_{i}\right)$;

$-d$-irrelevant ${ }^{\psi}\left(x_{i}\right) \rightarrow$ d-irrelevant ${ }^{\phi}\left(x_{i}\right)$;

$-\operatorname{dependent}^{\psi}\left(V, x_{i}\right) \rightarrow \operatorname{dependent}^{\phi}\left(V, x_{i}\right)$. 
Proof. All the results rely essentially on the fact that out $\subseteq$ sol. For the properties of inconsistency, implication, determinacy and dependence, the proof directly follows: classical inconsistency means that $\forall t \in$ sol. $t_{x_{i}} \neq a$, which implies the deep property $\forall t \in$ out. $t_{x_{i}} \neq a$; classical determinacy means that $\forall t \in$ sol. $\forall b \neq t_{x_{i}} . t\left[x_{i}:=b\right] \notin$ sol, which implies $\forall t \in$ out. $\forall b \neq t_{x_{i}} . t\left[x_{i}:=b\right] \notin$ sol, which implies the deep property $\forall t \in$ out. $\forall b \neq t_{x_{i}} . t\left[x_{i}:=b\right] \notin$ out. The cases of implication and dependence are similar.

For the other properties we additionally use Proposition 2: classical fixability means that $\forall t \in$ sol. $t\left[x_{i}:=a\right] \in$ sol. This implies $\forall t \in$ out. $t\left[x_{i}:=a\right] \in$ sol which, by Proposition 2, is equivalent to the deep property $\forall t \in$ out. $t\left[x_{i}:=a\right] \in$ out. The cases of substitutability, removability, interchangeability and irrelevance are similar.

Proposition 4. For all variables $x_{i}$ and values $a$ and $b$, we have:

$-d$-fixable $\left(x_{i}, a\right) \rightarrow$ s-fixable $\left(x_{i}, a\right) ;$

- d-removable $\left(x_{i}, a\right) \rightarrow$ s-removable $\left(x_{i}, a\right)$;

- d-substitutable $\left(x_{i}, a, b\right) \rightarrow$ s-substitutable $\left(x_{i}, a, b\right)$;

- d-interchangeable $\left(x_{i}, a, b\right) \rightarrow$ s-interchangeable $\left(x_{i}, a, b\right)$;

$-d$-irrelevant $\left(x_{i}\right) \rightarrow$ s-irrelevant $\left(x_{i}\right)$.

Proof. If deep fixability holds, i.e., we have $\forall t \in$ out. $t\left[x_{i}:=a\right] \in$ out, then for each $t \in$ out the tuple $t^{\prime}=t\left[x_{i}:=a\right]$ is such that $\left.t\right|_{X_{i-1}}=\left.t^{\prime}\right|_{X_{i-1}} \wedge t_{x_{i}}^{\prime}=a$, and we therefore have $\forall t \in$ out. $\exists t^{\prime} \in$ out. $\left(\left.t\right|_{X_{i-1}}=\left.t^{\prime}\right|_{X_{i-1}} \wedge t_{x_{i}}^{\prime}=a\right)$, which means s-fixable $\left(x_{i}, a\right)$. The proof is similar for irrelevance.

If deep removability holds, i.e., $\forall t \in$ out. $\left(t_{x_{i}}=a\right) \rightarrow\left(\exists b \neq a . t\left[x_{i}:=b\right] \in\right.$ out $)$, then for each $t \in$ out such that $t_{x_{i}}=a$, the tuple $t^{\prime}=t\left[x_{i}:=b\right]$ is such that $\left.t\right|_{X_{i-1}}=\left.t^{\prime}\right|_{X_{i-1}} \wedge t_{x_{i}}^{\prime}=b$, and we have s-removable $\left(x_{i}, a\right)$. The proof is similar for substitutability, which also uses a bounded quantification, and the result follows for interchangeability.

Proposition 5. The following relations hold between the properties (forall $x_{i}$, $a$ and $b)$ :

(1) $\operatorname{inconsistent}\left(x_{i}, a\right) \rightarrow \forall b \in D_{x_{i}}$. d-substitutable $\left(x_{i}, a, b\right)$;

(2) $\operatorname{implied}\left(x_{i}, a\right) \leftrightarrow \forall b \in D_{x_{i}} \backslash\{a\}$. inconsistent $\left(x_{i}, b\right)$;

(3) $\operatorname{implied}\left(x_{i}, a\right) \rightarrow d$-fixable $\left(x_{i}, a\right)$;

(4) $\operatorname{inconsistent}\left(x_{i}, a\right) \rightarrow d$-removable $\left(x_{i}, a\right)$;

(5) $\exists b \in D_{x_{i}} \backslash\{a\}$. d-substitutable $\left(x_{i}, a, b\right) \rightarrow$ d-removable $\left(x_{i}, a\right)$;

(6) $\exists b \in D_{x_{i}} \backslash\{a\}$. s-substitutable $\left(x_{i}, a, b\right) \rightarrow$ s-removable $\left(x_{i}, a\right)$;

(7) d-fixable $\left(x_{i}, b\right) \leftrightarrow \forall a \in D_{x_{i}}$. d-substitutable $\left(x_{i}, a, b\right)$;

(8) s-fixable $\left(x_{i}, b\right) \leftrightarrow \forall a \in D_{x_{i}}$. s-substitutable $\left(x_{i}, a, b\right)$;

(9) d-irrelevant $\left(x_{i}\right) \leftrightarrow \forall a \in D_{x_{i}}$. d-fixable $\left(x_{i}, a\right)$;

(10) s-irrelevant $\left(x_{i}\right) \leftrightarrow \forall a \in D_{x_{i}}$. s-fixable $\left(x_{i}, a\right)$.

Proof. (1) Assume inconsistency holds. If we consider an arbitrary $t \in$ out, then $t_{x_{i}} \neq a$, which falsifies the left side of the implication $\left(t_{x_{i}}=a\right) \rightarrow\left(t\left[x_{i}:=b\right] \in\right.$ out $)$, for any $b$, and deep substitutability therefore holds. 
(2) If value $a$ is implied for $x_{i}$, i.e., $\forall t \in$ out. $t_{x_{i}}=a$, then for every value $b \neq a$ we have $\forall t \in$ out. $t_{x_{i}}=a \neq b$, i.e., $b$ is inconsistent. If all values $b \neq a$ are inconsistent, i.e., $\forall t \in$ out. $t_{x_{i}} \neq b$, then any $t \in$ out is such that $\forall b \neq a . t_{x_{i}} \neq b$ and $t_{x_{i}} \in D_{x_{i}}$, so $t_{x_{i}}=a$ i.e., $a$ is implied.

(3) If $a$ is implied for $x_{i}$, then any $t \in$ out is such that $t_{x_{i}}=a$, and we therefore have $t\left[x_{i}:=a\right]=t \in$ out.

(4) If $a$ is inconsistent for $x_{i}$, i.e., $\forall t \in$ out. $t_{x_{i}} \neq a$, then the left-hand side of the implication $\left(t_{x_{i}}=a\right) \rightarrow\left(\exists b \neq a . t\left[x_{i}:=b\right] \in\right.$ out $)$ is false for every $t \in$ out.

(5) If $a$ is deep-substitutable to a certain value $b \neq a$, then for every $t \in$ out verifying $t_{x_{i}}=a$ we have $t\left[x_{i}:=b\right] \in$ out. This implies $\exists b \neq a . t\left[x_{i}:=b\right] \in$ out.

(6) If $a$ is shallow-substitutable to a certain value $b \neq a$, then for every $t \in$ out verifying $t_{x_{i}}=a$, we have $\exists t^{\prime} \in$ out. $\left(\left(\left.t\right|_{X_{i-1}}=\left.t^{\prime}\right|_{X_{i-1}}\right) \wedge\left(t_{x_{i}}^{\prime}=b\right)\right)$. This implies $\exists t^{\prime} \in$ out. $\left(\left.t\right|_{X_{i-1}}=\left.t^{\prime}\right|_{X_{i-1}} \wedge t_{x_{i}}^{\prime} \neq a\right)$.

(7) If $b$ is deep-fixable for $x_{i}$, i.e., $\forall t \in$ out. $t\left[x_{i}:=b\right] \in$ out, then the right-hand side of the implication $\left(t_{x_{i}}=a\right) \rightarrow\left(t\left[x_{i}:=b\right] \in\right.$ out $)$ is true for all $t \in$ out.

(8) If $b$ is shallow-fixable for $x_{i}$ i.e., $\forall t \in$ out. $\exists t^{\prime} \in$ out. $\left(\left.t\right|_{X_{i-1}}=\left.t^{\prime}\right|_{X_{i-1}} \wedge t_{x_{i}}^{\prime}=b\right)$, then the right-hand side of the implication $t_{x_{i}}=a \rightarrow \exists t^{\prime} \in$ out. $\left(\left(\left.t\right|_{X_{i-1}}=\left.t^{\prime}\right|_{X_{i-1}}\right) \wedge\right.$ $\left(t_{x_{i}}^{\prime}=b\right)$ is true for all $t \in$ out.

(9) If $x_{i}$ is deep-irrelevant, i.e., $\forall t \in$ out. $\forall a \in D_{x_{i}} . t\left[x_{i}:=a\right] \in$ out, then for any $a \in D_{x_{i}}$ we have $\forall t \in$ out. $t\left[x_{i}:=a\right] \in$ out.

(10) If $x_{i}$ is shallow-irrelevant, i.e., $\forall t \in$ out. $\forall a \in D_{x_{i}} . \exists t^{\prime} \in$ out. $\left(\left.t\right|_{X_{i-1}}=\right.$ $\left.\left.t^{\prime}\right|_{X_{i-1}}\right) \wedge\left(t_{x_{i}}^{\prime}=a\right)$, then for any $a \in D_{x_{i}}$ we have $\forall t \in$ out. $\exists t^{\prime} \in$ out. $\left(\left.t\right|_{X_{i-1}}=\right.$ $\left.\left.t^{\prime}\right|_{X_{i-1}} \wedge t_{x_{i}}^{\prime}=a\right)$

Proposition 6. Let $\phi=\langle X, Q, D, C\rangle$ be a $Q C S P$ in which value $a \in D_{x_{i}}$ is shallow-removable for an existential variable $x_{i}$, and let $\phi^{\prime}$ denote the same QCSP in which value a is effectively removed (i.e., $\phi^{\prime}=\left\langle X, Q, D^{\prime}, C\right\rangle$ where $D_{x_{i}}^{\prime}=D_{x_{i}} \backslash\{a\}$ and $\left.D_{x_{j}}^{\prime}=D_{x_{j}}, \forall j \neq i\right)$. Then $\phi$ is true iff $\phi^{\prime}$ is true.

Proof. If $\phi^{\prime}$ has a winning strategy then the same strategy is also winning for $\phi$; having $\phi^{\prime}$ true therefore implies that $\phi$ is also true.

On the other hand, assume that $\phi$ has a winning strategy $s^{1}$. Since $a \in D_{x_{i}}$ is shallow-removable for $x_{i}$, we have:

$$
\forall t \in \text { out. } t_{x_{i}}=a \rightarrow \exists t^{\prime} \in \text { out. }\left(\left.t\right|_{X_{i-1}}=\left.t^{\prime}\right|_{X_{i-1}} \wedge t_{x_{i}}^{\prime} \neq a .\right)
$$

We show that if $s^{1}$ has a scenario $t \in \operatorname{sce}\left(s^{1}\right)$ such that $t_{x_{i}}=a$, then we can "correct" this and exhibit another winning strategy $s$ whose scenarios are the same as those of $s^{1}$ except that all scenarios $\lambda$ such that $\left.\lambda\right|_{X_{i-1}}=\left.t\right|_{X_{i-1}}$ have been replaced by tuples $t^{\prime}$ with $t_{x_{i}}^{\prime} \neq a$. (Intuitively we replace the "sub-tree" corresponding to the branch $\left.t\right|_{X_{i-1}}$ by a new branch which does not involve the choice $x_{i}=a$ anymore.) More precisely, every scenario $t^{\prime} \in \operatorname{sce}(s)$ will satisfy:

-If $\left.t^{\prime}\right|_{X_{i-1}} \neq\left. t\right|_{X_{i-1}}$ then $t^{\prime} \in \operatorname{sce}\left(s^{1}\right)$.

-If $\left.t^{\prime}\right|_{X_{i-1}}=\left.t\right|_{X_{i-1}}$ then $t_{x_{i}}^{\prime} \neq a$.

This will prove the result: in showing how to construct $s$ we show that, starting from any winning strategy $s^{1}$ containing a number $n>0$ of "incorrect" scenarios $t^{\prime}$ with $t_{x_{i}}^{\prime}=a$, we can always exhibit a winning strategy with at most $n-1$ such 
scenarios, and repeating the correction $n$ times we construct a winning strategy in which no tuple $t^{\prime}$ is such that $t_{x_{i}}^{\prime}=a$.

Let us now see how to construct $s$ starting from $s^{1}$. The outcome $t \in \operatorname{sce}\left(s^{1}\right)$ that needs to be replaced is such that $t_{x_{i}}=a$ and, using the shallow removability property, we conclude that there exists another outcome $\theta \in$ out such that $\left.\theta\right|_{X_{i-1}}=$ $\left.t\right|_{X_{i-1}} \wedge \theta_{x_{i}} \neq a$. This outcome belongs to at least one winning strategy. We choose one of these strategies, which we call $s^{2}$. To define the new strategy $s$ we must define the functions $s_{x_{j}}$, for each $x_{j} \in E$. These functions are defined as follows:

-if $j<i$ then $s_{x_{j}}$ is defined as $s_{x_{j}}^{1}$ (e.g., we follow the strategy $s^{1}$ for the first variables, until variable $x_{i}$, excluded);

-for the following variables, i.e., when $j \geq i$, we define the value of $s_{x_{j}}(\tau)$, for each $\tau \in \prod_{y \in A_{j-1}} D_{y}$, as follows:

-if $\left.\tau\right|_{X_{i-1}}=\left.t\right|_{X_{i-1}}$, then $s_{x_{j}}(\tau)=s_{x_{j}}^{2}(\tau)$;

-if $\left.\tau\right|_{X_{i-1}} \neq\left. t\right|_{X_{i-1}}$, then $s_{x_{j}}(\tau)=s_{x_{j}}^{1}(\tau)$;

The proof is completed by checking that every scenario $t^{\prime} \in \operatorname{sce}(s)$ satisfies the two desired properties:

-If $\left.t^{\prime}\right|_{X_{i-1}} \neq\left. t\right|_{X_{i-1}}$ then $t^{\prime} \in \operatorname{sce}\left(s^{1}\right)$, because, for each $x_{j} \in E$, we have $t_{x_{j}}^{\prime}=$ $s_{x_{j}}\left(\left.t^{\prime}\right|_{A_{j-1}}\right)=s_{x_{j}}^{1}\left(\left.t^{\prime}\right|_{A_{j-1}}\right)$ in this case.

-If $\left.t^{\prime}\right|_{X_{i-1}}=\left.t\right|_{X_{i-1}}$ then $t_{x_{i}}^{\prime} \neq a$, because $t_{x_{i}}^{\prime}=s_{x_{i}}\left(\left.t^{\prime}\right|_{A_{i-1}}\right)=s_{x_{i}}^{2}\left(\left.t^{\prime}\right|_{A_{i-1}}\right)=$ $s_{x_{i}}^{2}\left(\left.t\right|_{A_{i-1}}\right)=s_{x_{i}}^{2}\left(\left.\theta\right|_{A_{i-1}}\right)=\theta_{x_{i}} \neq a$.

Furthermore, every $t^{\prime} \in \operatorname{sce}(s)$ with $\left.t^{\prime}\right|_{X_{i-1}}=\left.t\right|_{X_{i-1}}$ belongs to sce $\left(s^{2}\right)$, and $s$ is therefore a winning strategy: $\operatorname{sce}(s) \subseteq\left(\operatorname{sce}\left(s^{1}\right) \cup \operatorname{sce}\left(s^{2}\right)\right) \subseteq$ out.

Proposition 7. Let $\phi=\langle X, Q, D, C\rangle$ be a QCSP in which value $a \in D_{x_{i}}$ is fixable for an existential variable $x_{i}$, and let $\phi^{\prime}$ denote the same QCSP in which value $a$ is effectively fixed (i.e., $\phi^{\prime}=\left\langle X, Q, D^{\prime}, C\right\rangle$ where $D_{x_{i}}^{\prime}=\{a\}$ and $D_{x_{j}}^{\prime}=$ $\left.D_{x_{j}}, \forall j \neq i\right)$. Then $\phi$ is true iff $\phi^{\prime}$ is true.

PROOF. If $\phi^{\prime}$ has a winning strategy then the same strategy is also winning for $\phi$; having $\phi^{\prime}$ true therefore implies that $\phi$ is also true.

On the other hand suppose that $\phi$ has a winning strategy $s^{1}$. That $a \in D_{x_{i}}$ is shallow-fixable for $x_{i}$ means that we have:

$$
\forall t \in \text { out. } \exists t^{\prime} \in \text { out. }\left(\left.t\right|_{X_{i-1}}=\left.t^{\prime}\right|_{X_{i-1}} \wedge t_{x_{i}}^{\prime}=a\right)
$$

The proof is similar to the one already detailed for Prop. 6: we show that if $s^{1}$ has a scenario $t \in \operatorname{sce}\left(s^{1}\right)$ such that $t_{x_{i}} \neq a$, then we can "correct" this and exhibit another winning strategy $s$ whose scenarios are the same as those of $s^{1}$ except that all scenarios $\lambda$ such that $\left.\lambda\right|_{X_{i-1}}=\left.t\right|_{X_{i-1}}$ have been replaced by tuples $t^{\prime}$ with $t_{x_{i}}^{\prime}=a$. More precisely, every scenario $t^{\prime} \in \operatorname{sce}(s)$ will satisfy:

-If $\left.t^{\prime}\right|_{X_{i-1}} \neq\left. t\right|_{X_{i-1}}$ then $t^{\prime} \in \operatorname{sce}\left(s^{1}\right)$.

-If $\left.t^{\prime}\right|_{X_{i-1}}=\left.t\right|_{X_{i-1}}$ then $t_{x_{i}}^{\prime}=a$.

This will prove the result: in showing how to construct $s$ we show that, starting from any winning strategy $s^{1}$ containing a number $n>0$ of "incorrect" scenarios $t^{\prime}$ with $t_{x_{i}}^{\prime} \neq a$, we can always exhibit a winning strategy with at most $n-1$ such 
scenarios. This shows that there exists a winning strategy in which no tuple $t^{\prime}$ is such that $t_{x_{i}}^{\prime} \neq a$.

Let us now see how to construct $s$ starting from $s^{1}$. The outcome $t \in \operatorname{sce}\left(s^{1}\right)$ needs to be replaced. Using the shallow fixability property, we know that there exists another outcome $\theta \in$ out such that $\left.\theta\right|_{X_{i-1}}=\left.t\right|_{X_{i-1}} \wedge \theta_{x_{i}}=a$. This outcome belongs to at least one winning strategy. We choose one of these strategies, which we call $s^{2}$. To define the new strategy $s$ we must define the functions $s_{x_{j}}$, for each $x_{j} \in E$. These functions are defined as follows:

-if $j<i$ then $s_{x_{j}}$ is defined as $s_{x_{j}}^{1}$ (e.g., we follow the strategy $s^{1}$ for the first variables, until variable $x_{i}$, excluded);

-for the following variables, i.e., when $j \geq i$, we define the value of $s_{x_{j}}(\tau)$, for each $\tau \in \prod_{y \in A_{j-1}} D_{y}$, as follows:

-if $\left.\tau\right|_{X_{i-1}}=\left.t\right|_{X_{i-1}}$, then $s_{x_{j}}(\tau)=s_{x_{j}}^{2}(\tau)$;

-if $\left.\tau\right|_{X_{i-1}} \neq\left. t\right|_{X_{i-1}}$, then $s_{x_{j}}(\tau)=s_{x_{j}}^{1}(\tau)$;

The proof is completed by checking that every scenario $t^{\prime} \in \operatorname{sce}(s)$ satisfies the two desired properties:

-If $\left.t^{\prime}\right|_{X_{i-1}} \neq\left. t\right|_{X_{i-1}}$ then $t^{\prime} \in \operatorname{sce}\left(s^{1}\right)$, because, for each $x_{j} \in E$, we have $t_{x_{j}}^{\prime}=$ $s_{x_{j}}\left(\left.t^{\prime}\right|_{A_{j-1}}\right)=s_{x_{j}}^{1}\left(\left.t^{\prime}\right|_{A_{j-1}}\right)$ in this case.

-If $\left.t^{\prime}\right|_{X_{i-1}}=\left.t\right|_{X_{i-1}}$ then $t_{x_{i}}^{\prime}=a$, because $t_{x_{i}}^{\prime}=s_{x_{i}}\left(\left.t^{\prime}\right|_{A_{i-1}}\right)=s_{x_{i}}^{2}\left(\left.t^{\prime}\right|_{A_{i-1}}\right)=$ $s_{x_{i}}^{2}\left(\left.t\right|_{A_{i-1}}\right)=s_{x_{i}}^{2}\left(\left.\theta\right|_{A_{i-1}}\right)=\theta_{x_{i}}=a$.

Furthermore, every $t^{\prime} \in \operatorname{sce}(s)$ with $\left.t^{\prime}\right|_{X_{i-1}}=\left.t\right|_{X_{i-1}}$ belongs to sce $\left(s^{2}\right)$, and $s$ is therefore a winning strategy: $\operatorname{sce}(s) \subseteq\left(\operatorname{sce}\left(s^{1}\right) \cup \operatorname{sce}\left(s^{2}\right)\right) \subseteq$ out.

Proposition 8. Let $\phi=\langle X, Q, D, C\rangle$ be a QCSP in which value a $\in D_{x_{i}}$ is dual-shallow-removable for a universal variable $x_{i}$, and let $\phi^{\prime}$ denote the same QCSP in which value a is effectively removed (i.e., $\phi^{\prime}=\left\langle X, Q, D^{\prime}, C\right\rangle$ where $D_{x_{i}}^{\prime}=D_{x_{i}} \backslash$ $\{a\}$ and $\left.D_{x_{j}}^{\prime}=D_{x_{j}}, \forall j \neq i\right)$. Then $\phi$ is true iff $\phi^{\prime}$ is true.

Proof. Direct consequence of Prop. 6: the hypothesis is that the dual-shallowremovability holds, i.e., $a$ is removable for $x_{i}$ w.r.t. the negated QCSP $\neg \phi$; then $\phi$ is true iff $\neg \phi$ is false iff $\neg \phi^{\prime}$ is false iff $\phi^{\prime}$ is true.

Proposition 9. Let $\phi=\langle X, Q, D, C\rangle$ be a QCSP in which value $a \in D_{x_{i}}$ is dual-shallow-fixable for an universal variable $x_{i}$, and let $\phi^{\prime}$ denote the same QCSP in which value a is effectively fixed (i.e., $\phi^{\prime}=\left\langle X, Q, D^{\prime}, C\right\rangle$ where $D_{x_{i}}^{\prime}=\{a\}$ and $\left.D_{x_{j}}^{\prime}=D_{x_{j}}, \forall j \neq i\right)$. Then $\phi$ is true iff $\phi^{\prime}$ is true.

Proof. Direct consequence of Prop. 7: the hypothesis is that the dual-shallowfixability holds, i.e., $a$ is fixable for $x_{i}$ w.r.t. the negated QCSP $\neg \phi$; then $\phi$ is true iff $\neg \phi$ is false iff $\neg \phi^{\prime}$ is false iff $\phi^{\prime}$ is true.

Proposition 10. Let $\phi=\langle X, Q, D, C\rangle$ be a QCSP. Given a tuple $t \in \prod_{x \in X} D_{x}$, we denote by $B$ the conjunction of constraints:

$$
\bigwedge_{x_{i} \in E}\left(\left(\bigwedge_{y \in A_{i-1}} y=t_{y}\right) \rightarrow\left(x_{i}=t_{x_{i}}\right)\right)
$$

ACM Transactions on Computational Logic, Vol. V, No. N, October 2018. 
The $Q C S P \psi=\langle X, Q, D, B \cup C\rangle$ is true iff $t \in$ out $^{\phi}$.

Proof. Assume that $\psi$ is true. Then it has a non empty set of winning strategies; let $s$ be one of them, picked arbitrarily. Let $t^{\prime}$ be the scenario of $s$ that is such that $\left.t^{\prime}\right|_{A}=\left.t\right|_{A}$, i.e., that assigns the same values as $t$ on the universal variables. Because $s$ is a winning strategy, $t^{\prime}$ is a solution, and it satisfies the constraint given by (4). A straightforward induction on the indices of the existential variables shows that $t$ is indeed identical to $t^{\prime}$, which implies $t \in$ out $^{\phi}$.

Assume now that $t \in$ out $^{\phi}$, i.e., there exists a winning strategy $s$ for $\phi$ such that $t \in \operatorname{sce}(s)$. Every scenario $t^{\prime} \in \operatorname{sce}(s)$ satisfies $C$. Let us prove by case that each $t^{\prime} \in \operatorname{sce}(s)$ also satisfies $B$. If we consider the scenario $t^{\prime}$ which is such that $\left.t^{\prime}\right|_{A}=\left.t\right|_{A}$, then this scenario is indeed $t$ (a strategy defines a unique outcome for each assignment of the universal variables), which satisfies $B$. On the other hand, $B$ is satisfied also if we consider any tuple $t^{\prime}$ which is such that $\left.t^{\prime}\right|_{A} \neq\left. t\right|_{A}$. To see this, let $j$ be the lowest index such that $\left.t^{\prime}\right|_{A_{j-1}} \neq\left. t\right|_{A_{j-1}}$. Constraints of $B$ with $i<j$ are satisfied because $\left.t^{\prime}\right|_{A_{i-1}}=\left.t\right|_{A_{i-1}}$; the others because the left-hand side of the implications $\left(\bigwedge_{y \in A_{i-1}} t_{y}^{\prime}=t_{y}\right) \rightarrow\left(t_{x_{i}}^{\prime}=t_{x_{i}}\right)$ are false. Every scenario of $s$ therefore satisfies $B \wedge C$, in other words this strategy is winning for $\psi$.

Proposition 11. Given a QCSP $\phi=\langle X, Q, D, C\rangle$, the problems of deciding whether:

-value $a \in D_{x_{i}}$ is d-fixable, $d$-removable, inconsistent, implied for variable $x_{i} \in$ $X$,

-value $a \in D_{x_{i}}$ is $d$-substitutable to or $d$-interchangeable with $b \in D_{x_{i}}$ for variable $x_{i} \in X$,

—variable $x_{i} \in X$ is dependent on variables $V \subseteq X$, or is d-irrelevant,

are PSPACE-complete.

Proof. (membership in PSPACE) The membership in PSPACE relies essentially on Prop. 10 and its immediate consequence, mentioned in the main text, that testing whether $t \in$ out can be done in polynomial space. All properties hold iff some statement is verified for all $t \in$ out, so the idea is then to loop over each tuple $t$, determine whether it belongs to out and, if this is the case, check whether it satisfies the statement. For inconsistency we check whether $t_{x_{i}} \neq a$. We return false as soon as we have a tuple $t \in$ out for which this is not the case. For implication we test whether $t_{x_{i}}=a$ and similarly return false if one tuple does not verify that. The same idea works for all properties: for fixability we test whether $t\left[x_{i}:=a\right] \in$ out; for substitutability we check whether $\left(t_{x_{i}}=a\right) \rightarrow\left(t\left[x_{i}:=b\right] \in\right.$ out $)$; for removability we check whether $\left(t_{x_{i}}=a\right) \rightarrow\left(\exists b \neq a . t\left[x_{i}:=b\right] \in\right.$ out $)$; for determinacy we check whether $\forall b \neq t_{x_{i}} . t\left[x_{i}:=b\right] \notin$ out; for irrelevance we check whether $\forall b \in D_{x_{i}} . t\left[x_{i}:=b\right] \in$ out. For dependency we have to do a double loop in lexicographical order, check whether both tuples $t, t^{\prime}$ belong to out and, if, so, check whether $\left(\forall x_{j} \in V \cdot t_{x_{j}}=t_{x_{j}}^{\prime}\right) \rightarrow\left(t_{x_{i}}=t_{x_{i}}^{\prime}\right)$. In any case, at the end of the loop, we return true if no counter-example to the property has been found. It is clear that these algorithms use polynomial space and return true iff the considered property holds. 
Proof. (hardness for PSPACE) For all properties we reduce the problem of deciding whether a QCSP $\phi=\langle X, Q, D, C\rangle$ is false to the problem of testing whether the considered property holds.

The reductions work as follows. For inconsistency we simply construct the QCSP $\left.\psi=\left\langle X \cup\{x\}, Q^{\prime}, D^{\prime}, C\right\}\right\rangle$, where:

$-x$ is a fresh variable, i.e., $x \notin X$;

$-Q^{\prime}$ is similar to $Q$ except that the new variable $x$ is quantified existentially, i.e., $Q_{y}^{\prime}=Q_{y}, \forall y \neq x$ and $Q_{x}^{\prime}=\exists$;

$-D^{\prime}$ is similar to $D$ except that the domain of the new variable $x$ is a singleton, i.e., $D_{y}^{\prime}=D_{y}, \forall y \neq x$ and $D_{x}^{\prime}=\{a\}$ for some arbitrary $a$.

It is straightforward that $\phi$ has a winning strategy iff $\psi$ also does. Let us verify that $\phi$ is false iff value $a$ is inconsistent for variable $x$ in $\psi$ : if $\phi$ is false then out ${ }^{\phi}$ is empty, and so is out ${ }^{\psi}$, and then it is true that $\forall t \in$ out $^{\psi} . t_{x_{i}} \neq a$; if $a$ is inconsistent for $x$ in $\psi$ then $\forall t \in$ out $^{\psi} . t_{x_{i}} \neq a$, but no outcome can assign a value different from $a$ to variable $x_{i}$, hence out ${ }^{\psi}$ is empty and out ${ }^{\phi}$ is also empty.

The same reduction works directly for removability: $\phi$ is false iff $a$ is removable from $x$ in $\psi$.

For fixability, implication, substitutability, interchangeability and irrelevance, the reduction is only slightly different; now we construct the QCSP:

$$
\left.\psi=\left\langle X \cup\{x\}, Q^{\prime}, D^{\prime}, C \cup\{x=0\}\right\}\right\rangle
$$

in which the new variable $x$ is existential and ranges over $\{0,1\}$. Note that the constraint $x=0$ can be expressed directly in each and every of our 5 formalisms. We can check that $\phi$ is false iff:

- variable $x$ is fixable to value 1 in $\psi$ : if $\phi$ is false then out ${ }^{\phi}$ is empty and so is out $^{\psi}$ and we trivially have $\forall t \in$ out $^{\psi} . t[x:=1] \in$ out $^{\psi}$; if $x$ is fixable to 1 in $\psi$ then $\forall t \in$ out $^{\psi} . t[x:=1] \in$ out $^{\psi}$, but there is no $t$ is such that $t[x:=1] \in$ out $^{\psi}$ and out ${ }^{\psi}$ and out ${ }^{\phi}$ are empty.

- value 1 is implied for variable $x$ in $\psi$ : similarly to fixability we have out ${ }^{\phi}=\emptyset$ iff $\forall t \in$ out $^{\psi} \cdot t_{x}=1$.

—value 0 is substitutable to value 1 for variable $x$ in $\psi$ (out ${ }^{\phi}=\emptyset$ holds iff $\forall t \in$ out $^{\psi}$. $\left(t_{x}=0\right) \rightarrow\left(t[x:=1] \in\right.$ out $\left.\left.^{\psi}\right)\right)$.

- value 0 is interchangeable with value 1 for variable $x$ in $\psi$ : (out ${ }^{\phi}=\emptyset$ holds iff $\forall t \in$ out $^{\psi} .\left(t_{x}=0\right) \leftrightarrow\left(t[x:=1] \in\right.$ out $\left.\left.^{\psi}\right)\right)$.

-variable $x$ is irrelevant in $\psi:$ if $\forall t \in$ out $^{\psi} . \forall b \in\{0,1\} . t[x:=b] \in$ out $^{\psi}$, then any $t \in$ out $^{\psi}$ is in particular such that $t[x:=1] \in$ out $^{\psi}$ so no such $t$ exists and out $^{\psi}=\emptyset$. (The other direction is trivial.)

For determinacy and dependence, the reduction consists in constructing the QCSP $\left.\psi=\left\langle X \cup\{x\}, Q^{\prime}, D^{\prime}, C\right\}\right\rangle$, in which the new variable $x$ is existential and ranges over $\{0,1\}$.

We check that $\phi$ is false if $x$ is determined in $\psi$. Assume that $\forall t \in$ out $^{\psi} . \forall b \neq$ $t_{x} . t[x:=b] \notin$ out $^{\psi}$, and let us consider an arbitrary $t \in$ out $^{\psi}$. Its value on $x$ is either 0 or 1 (say 0 ). Then it is such that $t[x:=1] \notin$ out $^{\psi}$. Because values 0 and 
1 play a symmetric role, this cannot be, and out ${ }^{\phi}=\emptyset$. (The other implication is trivial.)

We last check that $\phi$ is false if variable $x$ is dependent on the set of variables $X$ in $\psi$. Assume that $\forall t, t^{\prime} \in$ out. $\left(\left.t\right|_{X}=\left.t^{\prime}\right|_{X}\right) \rightarrow\left(t_{x}=t_{x}^{\prime}\right)$. Let us consider an arbitrary tuple $t \in$ out $^{\psi}$ with (say) $t_{x}=0$. If we consider the tuple $t^{\prime}=t[x:=1]$, then this tuple is such that $\left.t^{\prime}\right|_{X}=\left.t\right|_{X}$, and therefore does not belong to out ${ }^{\psi}$ (if it did, then we'd have $t_{x}^{\prime}=t_{x}$ ). Because values 0 and 1 play a symmetric role, this cannot be, and out ${ }^{\phi}=\emptyset$. (The other implication is trivial.)

In all our reductions, we can start from any of the 5 formalisms listed in Sec. 6.1, and the resulting QCSP is expressed in the same formalism. It is well-known that deciding the truth of a QCSP in any of these formalisms is PSPACE-complete and the hardness result therefore holds in all 5 cases.

Proposition 12. Given a QCSP $\phi=\langle X, Q, D, C\rangle$, the problems of deciding whether:

-value $a \in D_{x_{i}}$ is s-fixable, s-removable for variable $x_{i} \in X$,

-value $a \in D_{x_{i}}$ is s-substitutable to or s-interchangeable with $b \in D_{x_{i}}$ for variable $x_{i} \in X$,

-variable $x_{i} \in X$ is s-irrelevant,

are PSPACE-complete.

Proof. For membership in PSPACE the algorithm is similar to Prop. 11: we use the fact that testing whether $t \in$ out can be done in polynomial space by Prop. 10. To check whether a property of the form $\forall t \in$ out. $\gamma$ is true, we loop over all tuples in lexicographical order, test whether the current tuple is an outcome and, if so, verify that it satisfies $\gamma$. For properties of the form $\exists t \in$ out. $\gamma$, we do a similar loop and return true iff one of the outcomes met during the loop satisfied $\gamma$. This works in polynomial space for all properties.

The hardness is a direct consequence of the fact that shallow properties are equivalent to the deep ones in the particular case when the variable on which the property is asserted is at the tail of the linearly ordered set of variables. In all the reductions used in the proof of Prop. 11, note that we introduce a variable that can be introduced at an arbitrary place. The reductions can therefore be directly adapted to the shallow definitions.

For instance, in the case of fixability, the reduction consisted, starting from a QCSP $\phi=\langle X, Q, D, C\rangle$, to construct the QCSP $\psi=\left\langle X \cup\{x\}, Q^{\prime}, D^{\prime}, C \cup\{x=0\}\right\rangle$, with $D_{x}^{\prime}=\{0,1\}$. We consider the same reduction and impose that $x$ be placed at the end of the ordered set $X$. Then $x$ is shallow-fixable to 1 iff it is deep-fixable to 1. We have proved that $\phi$ is false if variable $x$ is deep-fixable to 1 in $\psi$, which is true if it is shallow-fixable to 1 in $\psi$. Similarly in all cases of Prop. 11 the reduction directly applies to shallow property as long as we impose that the new variable $x$ be put at the end of the quantifier prefix.

Proposition 13. Given a $\Sigma_{k} Q C S P \phi=\langle X, Q, D, C\rangle$ encoded using Formalism (I), the problems of deciding whether:

-value $a \in D_{x_{i}}$ is deep-fixable, deep-removable, inconsistent, implied for variable $x_{i} \in X$, 
App-10

-value $a \in D_{x_{i}}$ is deep-substitutable to or deep-interchangeable with $b \in D_{x_{i}}$ for variable $x_{i} \in X$,

-variable $x_{i} \in X$ is dependent on variables $V \subseteq X$, or is deep-irrelevant,

are $\Pi_{k}^{p}$-hard and belong to $\Pi_{k+1}^{p}$. Moreover, for deep inconsistency, implication, determinacy and dependence, the problems are more precisely $\Pi_{k}^{p}$-complete.

The use of formalism (1) means that $\phi$ is a Quantified Boolean Formula of the form:

$$
\phi: \quad \exists M_{1}, \forall M_{2}, \cdots Q_{k} M_{k} . C
$$

where the $M_{i}$ s are blocks of variables of alternating quantification, $C$ is a Boolean circuit built on these variables, and the last block $M_{k}$ is quantified universally $\left(Q_{k}=\forall\right)$ if $k$ is even, and existentially $\left(Q_{k}=\exists\right)$ if $k$ is odd. Consistently with previous notation, the linearly ordered set $X=\left\{x_{1} \ldots x_{n}\right\}$ denotes the union of all variables of the prefix, and the notations $E_{j}, A_{j}$, etc., are defined as in Section 2.1.

For technical reasons it is more convenient to analyze the complexity of the negations of these properties, i.e., we focus on the complexity of determining whether the property does not hold. So we prove that the negations are $\Sigma_{k}^{p}$-hard and belong to $\Sigma_{k+1}^{p}$. (The problem of testing whether a $\Sigma_{k}^{p} \mathrm{QBF}$ is false is $\Pi_{k}^{p}$-complete.)

Proof. (membership results) For consistency, membership in $\Sigma_{k}^{p}$ is shown as follows: we are given a formula $\phi$ of the aforementioned form, as well as $a$ and $x_{i}$, and we want to test whether $\exists t \in$ out $^{\phi} . t_{x_{i}}=a$. We use a reduction similar to the one used by Prop. 10, and construct a formula which is true iff the property holds. The formula used in Prop. 10 imposes additional constraints whose role is to make sure that the outcome belongs to the set of scenarios of any winning strategy of the produced formula. In our case the outcome in question is quantified existentially and is of the form $\left\langle v_{1}, \ldots, v_{n}\right\rangle$ with $v_{i}=a$. We obtain the formula:

$$
\psi: \quad \exists v_{1}, \ldots v_{n} . \exists M_{1}, \forall M_{2} \cdots Q_{k} M_{k} .\left(B \wedge C \wedge v_{i}=a\right)
$$

where each variable $v_{i}$ ranges over $D_{x_{i}}$ and $B$ is the conjunction:

$$
\bigwedge_{x_{i} \in E}\left(\left(\bigwedge_{y_{j} \in A_{i-1}} y_{j}=v_{j}\right) \rightarrow\left(x_{i}=v_{i}\right)\right)
$$

Note that the existentially quantified variables $\left\langle v_{1}, \ldots, v_{n}\right\rangle$ are not redundant with the $x_{j}$ s: we want to impose that at least one of the outcomes of $\psi$ assign $x_{i}$ to $a$, whereas simply adding the constraint $x_{i}=a$ would enforce it for every scenario of any strategy. Formula $\psi$ is true iff there exists a tuple $t \in$ out $^{\phi}$ such that $t_{x_{i}}=a$ is a direct consequence of Prop. 10. Formula $\psi$ is itself a $\Sigma_{k}$-QBF and we can therefore determine whether it is true in $\Sigma_{k}^{p}$.

Non-implication $\left(\exists t \in\right.$ out. $\left.t_{x_{i}} \neq a\right)$, Eq. 5 is simply replaced by:

$$
\psi: \quad \exists v_{1}, \ldots v_{n} . \exists M_{1} . \forall M_{2} \cdots Q_{k} M_{k} .\left(B \wedge C \wedge v_{i} \neq a\right)
$$

Non-determinacy is expressed as $\exists t \in$ out. $\exists b \neq t_{x_{i}} . t\left[x_{i}:=b\right] \in$ out or, equivalently, as $\exists t \in$ out. $\exists t^{\prime} \in$ out. $\left.t^{\prime}\right|_{X \backslash\left\{x_{i}\right\}}=\left.t\right|_{X \backslash\left\{x_{i}\right\}} \wedge t_{x_{i}}^{\prime} \neq t_{x_{i}}$. We have to 
assert the joint existence of the two outcomes $t$ and $t^{\prime}$, whose values on variables $x_{1} \ldots x_{n}$ are noted $\left\langle v_{1}, \ldots, v_{n}\right\rangle$ and $\left\langle v_{1}^{\prime}, \ldots, v_{n}^{\prime}\right\rangle$, respectively. We obtain:

$$
\psi: \exists v_{1}, \ldots v_{n} . \exists v_{1}^{\prime}, \ldots v_{n}^{\prime} .\left(\begin{array}{c}
\bigwedge_{j \neq i} v_{j}^{\prime}=v_{j} \wedge v_{i}^{\prime} \neq v_{i} \\
\wedge \exists M_{1} \cdot \forall M_{2} \cdots Q_{k} M_{k} .(B \wedge C) \\
\wedge \exists M_{1}^{\prime} . \forall M_{2}^{\prime} \cdots Q_{k}^{\prime} M_{k}^{\prime} .\left(B^{\prime} \wedge C^{\prime}\right)
\end{array}\right)
$$

Now we note that the two matrices $(B \wedge C)$ and $\left(B^{\prime} \wedge C^{\prime}\right)$ are imposed on disjoint sets of variables (the unprimed and the primes variables, respectively), so we can rewrite the previous formula in a $\Sigma_{k}$ form, as follows:

$$
\psi: \exists v_{1}, \ldots v_{n} \cdot \exists v_{1}^{\prime}, \ldots v_{n}^{\prime} \cdot\left(\begin{array}{c}
\bigwedge_{j \neq i} v_{j}^{\prime}=v_{j} \wedge v_{i}^{\prime} \neq v_{i} \wedge \\
\exists M_{1}, M_{1}^{\prime} \cdot \forall M_{2}, M_{2}^{\prime} \\
\cdots Q_{k} M_{k}, M_{k}^{\prime} .\left(B \wedge C \wedge B^{\prime} \wedge C^{\prime}\right)
\end{array}\right)
$$

Non-dependence can be stated as $\exists t \in$ out. $\exists t^{\prime} \in$ out. $\left(\left.t\right|_{V}=\left.t^{\prime}\right|_{V}\right) \wedge\left(t_{x_{i}} \neq t_{x_{i}}^{\prime}\right)$, relying on the fact that the domain only has two values; the proof is similar except that $\psi$ has the following form:

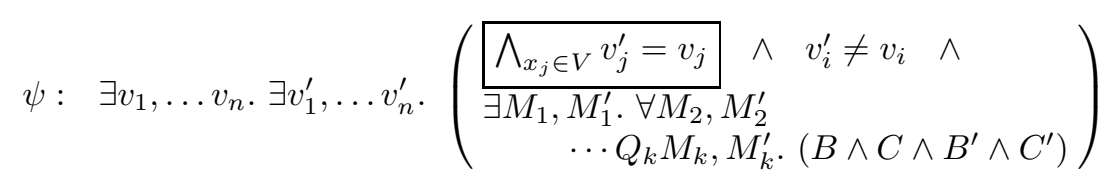

For the other properties it is less obvious to see whether the upper bound of $\Sigma_{k}^{p}$ holds, because their negations are defined as follows:

- Non-fixability can be expressed as $\exists t \in$ out. $t\left[x_{i}:=a\right] \notin$ out or, equivalently, $\exists t \in$ out. $\exists t^{\prime} \notin$ out. $\left.t^{\prime}\right|_{X \backslash\left\{x_{i}\right\}}=\left.t\right|_{X \backslash\left\{x_{i}\right\}} \wedge t_{x_{i}}^{\prime}=a$;

-Non-substitutability as $\exists t \in$ out. $\left(t_{x_{i}}=a\right) \wedge\left(t\left[x_{i}:=b\right] \notin\right.$ out $)$ or, equivalently, $\exists t \in$ out. $\exists t^{\prime} \notin$ out. $\left.t^{\prime}\right|_{X \backslash\left\{x_{i}\right\}}=\left.t\right|_{X \backslash\left\{x_{i}\right\}} \wedge t_{x_{i}}=a \wedge t_{x_{i}}^{\prime}=b$;

-Non-removability as $\exists t \in$ out. $\left(t_{x_{i}}=a\right) \wedge\left(\forall b \neq a . t\left[x_{i}:=b\right] \notin\right.$ out $)$ or, equivalently, as $\exists t \in$ out. $\forall t^{\prime} \notin$ out. $\left(\left.t^{\prime}\right|_{X \backslash\left\{x_{i}\right\}}=\left.t\right|_{X \backslash\left\{x_{i}\right\}}\right) \rightarrow t_{x_{i}}=t_{x_{i}}^{\prime}$;

- Non-irrelevance is expressed as $\exists t \in$ out. $\exists t^{\prime} \notin$ out. $\left.t^{\prime}\right|_{X \backslash\left\{x_{i}\right\}}=\left.t\right|_{X \backslash\left\{x_{i}\right\}}$.

The problem is that in each case we need to find both an outcome $t$ and another tuple $t^{\prime}$ which is not an outcome. The quantifier pattern for asserting that $t$ is not an outcome is now of the form $\forall M_{1} . \exists M_{2} \cdots \overline{Q_{k}} M_{k}$, where $\overline{Q_{k}}$ is the dual quantifier to $Q_{k}$. For instance for non-irrelevance the obtained formula has the following form:

$$
\exists v_{1}, \ldots v_{n} . \exists v_{1}^{\prime}, \ldots v_{n}^{\prime} \cdot\left(\begin{array}{c}
\bigwedge_{j \neq i} v_{j}^{\prime}=v_{j} \\
\wedge \exists M_{1} \cdot \forall M_{2} \cdots Q_{k} M_{k} .(B \wedge C) \\
\wedge \forall M_{1}^{\prime} . \exists M_{2}^{\prime} \cdots \overline{Q_{k}} M_{k}^{\prime} .\left(\neg B^{\prime} \vee \neg C^{\prime}\right)
\end{array}\right)
$$

Similarly to before, the variables involved in the matrics $(B \wedge C)$ and $\left(\neg B^{\prime} \vee \neg C^{\prime}\right)$ are disjoint and we can merge them into one prefix. We rename the indexing of the primed blocks as follows:

$$
\exists v_{1}, \ldots v_{n} \cdot \exists v_{1}^{\prime}, \ldots v_{n}^{\prime} \cdot\left(\begin{array}{c}
\bigwedge_{j \neq i} v_{j}^{\prime}=v_{j} \\
\wedge \exists M_{1} \cdot \forall M_{2} \cdots Q_{k} M_{k} .(B \wedge C) \\
\wedge \forall M_{2}^{\prime} . \exists M_{3}^{\prime} \cdots Q_{k+1} M_{k+1}^{\prime} \cdot\left(\neg B^{\prime} \vee \neg C^{\prime}\right)
\end{array}\right)
$$


and obtain:

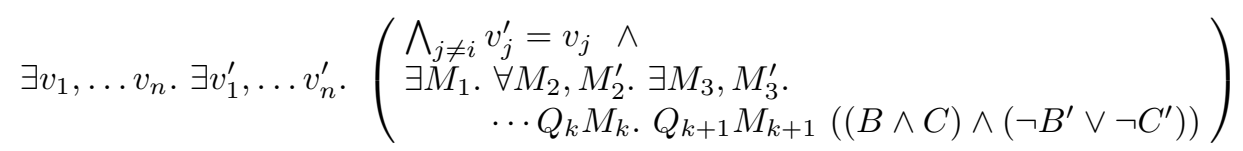

which is in $\Sigma_{k+1}^{p}$ form. We obtain a similar formula with minor changes for fixability, substitutability and removability.

Proof. (hardness) The hardness part is easy, because in all the reductions used in Prop. 11 to show the PSPACE-hardness of the properties, we reduced the problem of determining whether a QCSP $\phi$ is false to the problem of checking the considered property for a new formula $\psi$. The new formula $\psi$ was constructed by introducing a new existential variable and this variable could be added into any quantifier block. Because of that, we can always make sure that the quantifier prefix of $\psi$ follows the same alternation as the one of $\phi$, and we can therefore reduce the problem of determining whether a $\Sigma_{k}^{p} \mathrm{QBF}$ is false to the problem of testing the considered property is verified by a $\Sigma_{k}^{p} \mathrm{QBF}$.

For instance the reduction used to prove that inconsistency is PSPACE-complete was as follows: we reduced any QCSP $\phi: \exists M_{1} . \forall M_{2} . \cdots Q_{k} M_{k}$. C to the QCSP $\psi: \exists M_{1}^{\prime},\{x\} . \forall M_{2} . \cdots Q_{k} M_{k}$. $C$ with $D_{x}=\{a\}$. We had not specified the precise existential block in which the new variable $x$ was added because the proof was precisely independent of that. We can now impose that it be inserted in the first block $M_{1}$. This shows that we can reduce the problem of falsity for $\Sigma_{k}^{p} \mathrm{QBFs}$ to the problem of inconsistency for $\Sigma_{k}^{p}$ QBFs. Similarly, all the other proofs can be directly adapted to bounded quantifier alternations.

Proposition 14. Let $\phi=\langle X, Q, D, C\rangle$ be a QCSP where $C=\left\{c_{1}, \ldots, c_{m}\right\}$. We denote by $\phi_{k}$ the $Q C S P\left\langle X, Q, D,\left\{c_{k}\right\}\right\rangle$ in which only the $k$-th constraint is considered. We have, for all $x_{i} \in X, V \subseteq X$, and $a, b \in D_{x_{i}}$ :

$-\left(\bigvee_{k \in 1 . . m}\right.$ inconsistent $\left.^{\phi_{k}}\left(x_{i}, a\right)\right) \rightarrow$ inconsistent $^{\phi}\left(x_{i}, a\right)$;

$-\left(\bigvee_{k \in 1 . . m} \operatorname{implied}^{\phi_{k}}\left(x_{i}, a\right)\right) \rightarrow \operatorname{implied}^{\phi}\left(x_{i}, a\right)$;

$-\left(\bigwedge_{k \in 1 . . m} d\right.$-fixable $\left.{ }^{\phi_{k}}\left(x_{i}, a\right)\right) \rightarrow$ d-fixable ${ }^{\phi}\left(x_{i}, a\right)$;

$-\left(\bigwedge_{k \in 1 . . m} d\right.$-substitutable $\left.{ }^{\phi_{k}}\left(x_{i}, a, b\right)\right) \rightarrow d$-substitutable ${ }^{\phi}\left(x_{i}, a, b\right)$;

$-\left(\bigwedge_{k \in 1 . . m} d\right.$-interchangeable $\left.{ }^{\phi_{k}}\left(x_{i}, a, b\right)\right) \rightarrow d$-interchangeable ${ }^{\phi}\left(x_{i}, a, b\right)$;

$-\left(\bigvee_{k \in 1 . . m}\right.$ determined $\left.^{\phi_{k}}\left(x_{i}\right)\right) \rightarrow$ determined $^{\phi}\left(x_{i}\right)$;

$-\left(\bigwedge_{k \in 1 . . m} d\right.$-irrelevant $\left.{ }^{\phi_{k}}\left(x_{i}\right)\right) \rightarrow$ d-irrelevant ${ }^{\phi}\left(x_{i}\right)$;

$-\left(\bigvee_{k \in 1 . . m}\right.$ dependent $\left.^{\phi_{k}}\left(V, x_{i}\right)\right) \rightarrow \operatorname{dependent}^{\phi}\left(V, x_{i}\right)$.

Proof. These propositions rely on the following monotonicity property of the set of outcomes: if we have two QCSPs $\phi_{1}=\left\langle X, Q, D, C_{1}\right\rangle$ and $\phi_{2}=\left\langle X, Q, D, C_{2}\right\rangle$ (with the same quantifier prefix) and if sol ${ }^{\phi_{1}} \subseteq$ sol $^{\phi_{2}}$ then out ${ }^{\phi_{1}} \subseteq$ out $^{\phi_{2}}$. This is easy to see: any winning strategy $s$ for $\phi_{1}$ is such that $\operatorname{sce}(s) \subseteq$ sol $^{\phi_{1}}$. Then it is also such that $\operatorname{sce}(s) \subseteq$ sol $^{\phi_{2}}$ and it is a winning strategy for $\phi_{2}$.

The proofs for inconsistency, implication and determinacy directly follow: 
-For inconsistency: if for some $k$ we have $\forall t \in$ out $^{\phi_{k}} \cdot t_{x_{i}} \neq a$, then we also have $\forall t \in$ out $^{\phi} . t_{x_{i}} \neq a$, because out ${ }^{\phi} \subseteq$ out $^{\phi_{k}}$.

-For implication: if for some $k$ we have $\forall t \in$ out $^{\phi_{k}} \cdot t_{x_{i}}=a$, then we also have $\forall t \in$ out $^{\phi} . t_{x_{i}}=a$, because out ${ }^{\phi} \subseteq$ out $^{\phi_{k}}$.

-For determinacy: if for some $k$ we have $\forall t \in$ out $^{\phi_{k}} . \forall b \neq t_{x_{i}} . t\left[x_{i}:=b\right] \notin$ out $^{\phi_{k}}$, then we also have $\forall t \in$ out $^{\phi} . \forall b \neq t_{x_{i}} . t\left[x_{i}:=b\right] \notin$ out $^{\phi} \subseteq$ out $^{\phi_{k}}$.

- For dependence: if for some $k$ we have $\forall t, t^{\prime} \in$ out $^{\phi_{k}} .\left.t\right|_{V}=\left.t^{\prime}\right|_{V} \rightarrow t_{x_{i}}=t_{x_{i}}^{\prime}$, then we also have $\forall t, t^{\prime} \in$ out $^{\phi} .\left.t\right|_{V}=\left.t^{\prime}\right|_{V} \rightarrow t_{x_{i}}=t_{x_{i}}^{\prime}$ because out ${ }^{\phi} \subseteq$ out $^{\phi_{k}}$.

Consider now deep fixability. We assume that forall $k$ and forall $t \in$ out $^{\phi_{k}}$ we have $t\left[x_{i}:=a\right] \in$ out $^{\phi_{k}}$. We consider a tuple $t \in$ out $^{\phi}$; since out ${ }^{\phi} \subseteq$ out $^{\phi_{k}}$ for all $k, t$ belongs to every out ${ }^{\phi_{k}}$, and therefore $t\left[x_{i}:=a\right]$ belongs to every out ${ }^{\phi_{k}}$ and therefore to every sol $\phi_{k}$. We conclude that $t\left[x_{i}:=a\right] \in$ sol $^{\phi}=\bigcap_{k}$ sol $^{\phi_{k}}$. We have seen in Prop. 2 that deep fixability can be stated as $\forall t \in$ out $^{\phi} . t\left[x_{i}:=a\right] \in$ sol $^{\phi}$, which completes the proof.

For deep substitutability. We assume that forall $k$ and forall $t \in$ out $^{\phi_{k}}$ we have $t_{x_{i}}=a \rightarrow t\left[x_{i}:=b\right] \in$ out $^{\phi_{k}}$. We consider a tuple $t \in$ out $^{\phi}$ such that $t_{x_{i}}=a$; since out $^{\phi} \subseteq$ out $^{\phi_{k}}$ for all $k, t$ belongs to every out ${ }^{\phi_{k}}$, and therefore $t\left[x_{i}:=b\right]$ belongs to every out ${ }^{\phi_{k}}$ and therefore to every sol ${ }^{\phi_{k}}$. We conclude that $t\left[x_{i}:=b\right] \in$ sol $^{\phi}=$ $\bigcap_{k}$ sol $^{\phi_{k}}$. We have seen in Prop. 2 that deep substitutability can be stated as $\forall t \in$ out $^{\phi} . t_{x_{i}}=a \rightarrow t\left[x_{i}:=b\right] \in$ sol $^{\phi}$, which completes the proof.

For deep interchangeability the result follows since two values $a$ and $b$ are interchangeable iff $a$ is substitutable to $b$ and $b$ is substitutable to $a$.

For deep irrelevance we use a result of Prop. 5: variable $x_{i}$ is irrelevant iff it is fixable to any value $a \in D_{x_{i}}$. If forall $k$ we have $d$-irrelevant ${ }^{\phi_{k}}\left(x_{i}\right)$ then we have, forall $k$ and forall $a \in D_{x_{i}}$, d-fixable $e^{\phi_{k}}\left(x_{i}, a\right)$. It follows that, forall $a \in D_{x_{i}}$, $d$-fixable ${ }^{\phi}\left(x_{i}, a\right)$. This is equivalent to $d$-irrelevant ${ }^{\phi}\left(x_{i}\right)$. 